\title{
High Replicability of Newly-Discovered Social-behavioral Findings is Achievable
}

John Protzko*1,15, Jon Krosnick ${ }^{2}$, Leif Nelson ${ }^{4}$, Brian A. Nosek ${ }^{3,7}$, Jordan Axt ${ }^{5}$, Matt Berent ${ }^{6}$, Nicholas Buttrick ${ }^{7}$, Matthew DeBell ${ }^{2}$, Charles R. Ebersole ${ }^{7}$, Sebastian Lundmark ${ }^{9}$, Bo MacInnis $^{2}$, Michael O’Donnell ${ }^{10}$, Hannah Perfecto ${ }^{11}$, James E. Pustejovsky ${ }^{12}$, Scott Roeder ${ }^{13}$, Jan Walleczek $^{14}$, Jonathan W. Schooler ${ }^{1}$

${ }^{1}=$ University of California, Santa Barbara $;^{2}=$ Stanford University; ${ }^{3}=$ Center for Open Science ${ }^{4}=$ University of California, Berkeley; ${ }^{5}=$ McGill University; ${ }^{6}=$ Matt Berent Consulting; ${ }^{7}=$ University of Virginia $;{ }^{9}=$ University of Gothenburg; ${ }^{10}=$ Georgetown University; ${ }^{11}=$ Washington University in St. Louis; ${ }^{12}=$ University of Wisconsin - Madison; ${ }^{13}$ $=$ University of South Carolina $;{ }^{14}=$ Fetzer Franklin Fund $;{ }^{15}=$ Central Connecticut State University

* Corresponding Author: protzko@gmail.com 
Failures to replicate evidence of new discoveries have forced scientists to ask whether this unreliability is due to suboptimal implementation of methods or whether presumptively optimal methods are not, in fact, optimal. This paper reports an investigation by four coordinated laboratories of the prospective replicability of 16 novel experimental findings using rigorenhancing practices: confirmatory tests, large sample sizes, preregistration, and methodological transparency. In contrast to past systematic replication efforts that reported replication rates averaging 50\%, replication attempts here produced the expected effects with significance testing $(p<.05)$ in $86 \%$ of attempts, slightly exceeding maximum expected replicability based on observed effect sizes and sample sizes. When one lab attempted to replicate an effect discovered by another lab, the effect size in the replications was $97 \%$ that of the original study. This high replication rate justifies confidence in rigor enhancing methods to increase the replicability of new discoveries.

Keywords: Psychology; Marketing; Political Science; Judgment \& Decision-Making; Metascience; Open Science; Reproducibility 
Science progressively learns about the world through the discovery of replicable findings ${ }^{1-2}$. Efforts to systematically replicate studies across various scientific fields have reported seemingly-disappointing replication rates ranging from $30 \%$ to $70 \%$, with effect size(ES)s about half the size of original findings ${ }^{3-13}$. This has been a cause for concern among many-but not all ${ }^{14-15}$.

Seemingly low replicability can be the consequence of false positives or exaggerated ESs among the original studies, resulting from low statistical power, measurement problems, errors, p-hacking, and regression to the mean due to selective reporting favoring original positive results ${ }^{15-16}$. Low replicability can also be the consequence of false negatives or suppressed ESs among replication studies, resulting from sampling error, low statistical power, heterogeneity of the phenomena, different analytic strategies, questionable research practices, and/or lack of fidelity to the original protocols ${ }^{17-22}$. Finally, failures to replicate or declining ESs can be the consequence of unknown or unarticulated moderating influences and boundary conditions that differ between original and replication studies, indicating shortcomings in the theoretical and methodological specifications ${ }^{23}$.

Collectively, these factors comprise likely explanations for why replications are less successful and produce weaker ESs over time ${ }^{24-26}$. Here, we report the results of a prospective replication study examining whether low replicability and declining effects are inevitable when using proposed rigor-enhancing practices.

Four laboratories conducting discovery-oriented social-behavioral research participated in a prospective replication study. Over five years, the labs conducted their typical research, examining topics covering psychology, marketing, advertising, political science, communication, 
and judgment and decision-making (Table 1). Based on pilot experimentation, each of the four labs submitted four new candidate discoveries for a self-confirmatory test and four replications, for a total of 16 confirmatory tests and 64 replications. In the self-confirmatory test, the discovering lab conducted a preregistered study with a large sample $(\mathrm{N} \gtrsim 1500)$ and shared a report of the methodology. Regardless of the outcome of the self-confirmatory test, in the replication phase, all labs conducted independent preregistered replications using the written methodology and any specialized study materials shared by the discovering lab (e.g., videos constructed for delivering interventions). Ordinarily, we would promote strong communication between labs to maximize sharing of tacit knowledge about the methodology, but in this case, to maintain the independence of each replication, we opted to discourage communication with the discovering lab outside of the documented protocols except for critical methodology clarifications (see SI). The replicating labs used equally large sample sizes (N's $\gtrsim 1500$ ) and each lab used a different sample provider.

Preregistration, reporting all outcomes, large sample sizes, transparent archiving, and sharing of materials, and commitment to high fidelity replication procedures should minimize irreplicability or declining effect sizes stemming from questionable research practices, selective reporting, low-powered research, or poorly implemented replication procedures. Such optimizing might promote higher replicability than previously reported in the literature. If-despite these rigor-enhancing practices_-low replicability rates or declining effects are observed—such rates and declines could be intrinsic to social-behavioral scientific investigation ${ }^{40-43}$.

Each of the 16 ostensible discoveries were obtained through pilot and exploratory research conducted independently in each laboratory. Labs, using their own criteria, decided which ostensible discoveries to submit for a confirmatory test and replication. Labs introduced 4 
provisional discoveries each, resulting in 16 self-confirmatory tests and 64 replications ( 3 independent and 1 self-replication for each), testing replicability and decline. All confirmatory tests, replications, and analyses were preregistered in both the individual studies (see Table S2) and for this meta-project (https://osf.io/6t9vm).

\section{Results}

\section{Replicability Rate}

\section{Self-Confirmatory Tests}

$81 \%(13 / 16)$ of the self-confirmatory tests produced statistically-significant results $(\underline{d}=0.265$ (95\% CI $=0.165-0.37$; with $0.18 \mathrm{SD}$ estimated between-study heterogeneity). The average ES of the self-confirmatory tests was smaller than the estimated average ES of the published psychological literature $(\underline{d}=0.43)^{27}$, even when only considering the 13 statisticallysignificant findings $(\underline{d}=0.32)$. No lab produced self-confirmatory tests with larger average ESs than the other labs (robust Approximate Hotelling's $T^{2}(6.01)=0.6, p=.638$ ).

\section{Replications}

One way of assessing replicability is to examine whether each replication rejects the null hypothesis at $p<.05$ in the expected direction ${ }^{6}$. Including all 16 self-confirmatory tests, 55/64 (86\%) replications were successful. The average ES of the replications was $\underline{d}=0.26$ (95\% CI $=0.19-0.33)$, similar to the ES observed in the self-confirmatory tests $(\underline{d}=0.265)$ yet larger than replication ESs observed in prior attempts to systematically replicate the established social-behavioral literature $(\underline{d}=0.155)^{28}$. 
Focusing only on the 13 statistically-significant self-confirmatory tests, 47/52 (90\%) of the subsequent replications yielded statistically-significant effects in the hypothesized direction. The average ES of the replications was $\underline{d}=0.319$ (95\%CI=0.305-0.333), similar to the ES observed in the 13 self-confirmatory tests $(\underline{d}=0.324)$.

For the three statistically non-significant self-confirmatory tests, 8/12 (67\%) of the subsequent replications produced statistically-significant effects in the hypothesized direction: $1 / 4$ for one $(\mathbf{d}=0.03,95 \% \mathrm{CI}=-0.02-0.08) ; 3 / 4$ for the second $(\underline{d}=0.09,95 \% \mathrm{CI}=0.04-0.14)$; and $4 / 4$ for the third $(\underline{d}=0.15,95 \% \mathrm{CI}=0.1-0.2)$. These replication ESs were slightly larger than the ES observed in the original self-confirmatory tests $(\underline{d}=0.01 ;$ Fig 1$)$.

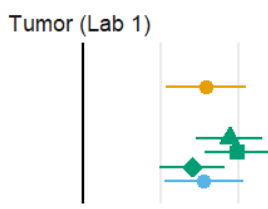

Minimal Groups (Lab 2)

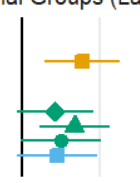

Cookies (Lab 3)

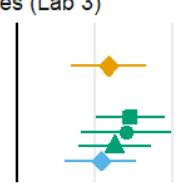

Labels (Lab 4)

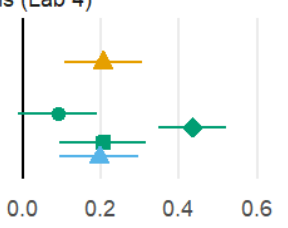

Confirmatory tes

Self-replication

Independent replication

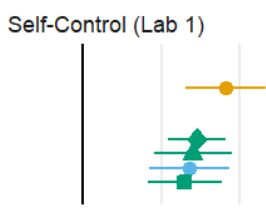

Orientation (Lab 2)

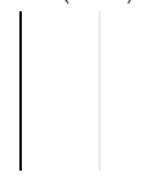

Referrals (Lab 3)
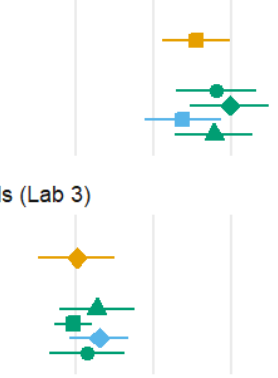

Ads (Lab 4)
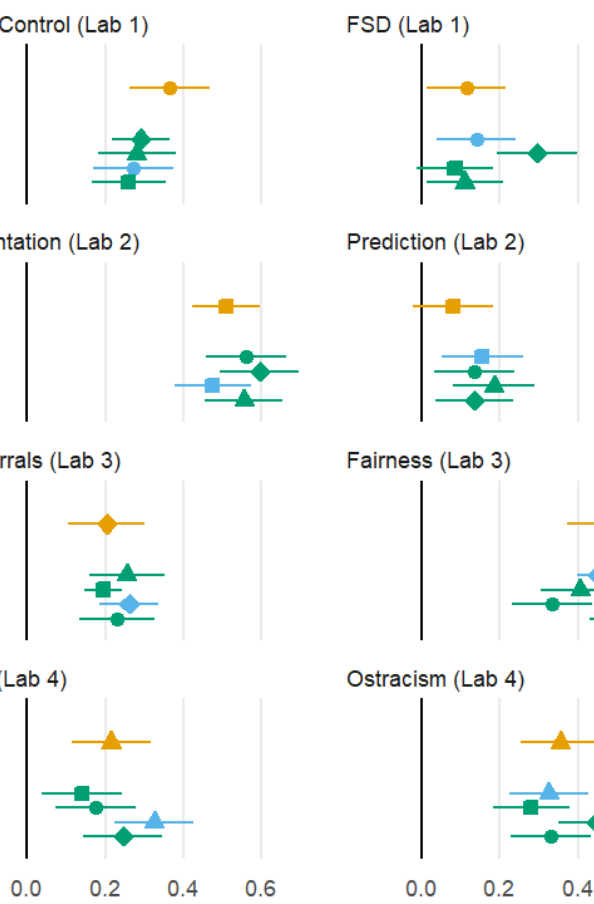

Prediction (Lab 2)

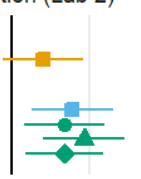

Fairness (Lab 3)

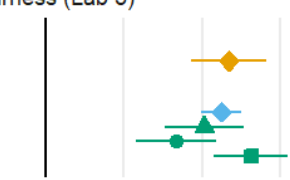

Ostracism (Lab 4)

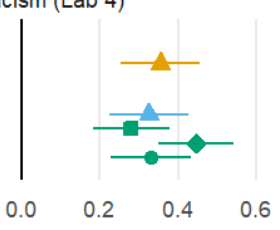

Effect size (Standardized mean difference)

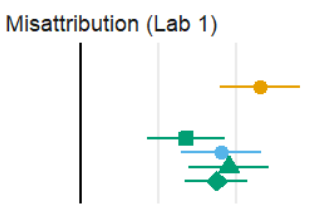

Redemption (Lab 2)

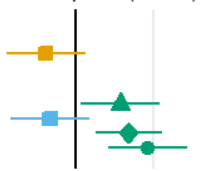

Worse (Lab 3)

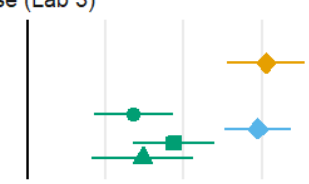

Misreporting (Lab 4)

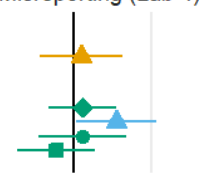

$\begin{array}{llll}0.0 & 0.2 & 0.4 & 0.6\end{array}$

Fig 1. Effect sizes and 95\%CI from 16 new discoveries (yellow marks) in the social-behavioral sciences with four replications each. Each lab is designated by a unique shape for observed effect size; blue marks correspond to self-replications, green marks to independent replications. 
An alternate index of replicability examines the consistency of ESs generated by the initial self-confirmatory test and its subsequent replications. Based on a multi-level metaanalysis, little variation in ESs was observed beyond what would be expected by sampling variation alone $\left(\hat{\tau}_{\text {within }}=0.06,95 \% \mathrm{CI}=0.04-0.08\right)$. This indicates the self- and independent replications would not have perfectly replicated the ES of the self-confirmatory tests, even if all samples were large enough to make sampling error negligible. The degree of variation was, however, smaller than the variation in ESs across the 16 discoveries $\left(\hat{\tau}_{\text {between }}=0.14\right.$, $95 \% \mathrm{CI}=0.1-0.2$ ). This suggests variation due to sampling and procedural differences across labs was much smaller than the variation due to the phenomena being investigated ${ }^{7-8}$.

In an exploratory model, differences between self-confirmatory tests and self-replication ESs were found to be fully attributable to sampling error, and average ESs in independent replications strongly correlated with ESs from self-confirmatory tests and self-replications $(r=.83$ 95\% CI=.52-.95). There was a small amount of heterogeneity across the independent replications $\left(\hat{\tau}_{\text {within }}=0.05,95 \% \mathrm{CI}=0.03-0.08\right)$. This suggests conducting replications in new samples and with independently implemented methods increased variability in the observed ESs, but did not systematically reduce the observed ESs.

Based on a power analysis of the 13 self-confirmatory tests with statistically-significant results, the average replication power was 0.96 with a median approaching 1 and average power in replication studies of specific discoveries ranging from 0.62 to approaching 1 . The observed replication rate of $90 \%$ is slightly smaller than expected based on these power estimates. Considering all self-confirmatory tests, including statistically non-significant ones, average power across attempted replications was .80 with a median of .99 and a range of 0 to approaching 1 . The observed replication rate of $86 \%$ in the replications for all 16 discoveries was 
somewhat larger than expected based on power estimates. Overall, replication rates were consistent with power estimates calculated using the ESs observed in self-confirmatory tests.

\section{Declines in Effect Sizes Across Replications}

Within-study heterogeneity across replications was estimated to 0.06SD, suggesting little heterogeneity overall, despite $75 \%$ of the replications being conducted independently using different sample providers. There was modest evidence that one lab produced slightly smaller ESs in replications compared to one other lab, controlling for the average size of original effects from each lab (robust Approximate Hotelling's $\left.T^{2}(12.31)=3.51, p=.048\right)$. The multi-level analyses lab used fixed effects.

When comparing the self-confirmatory tests to their self-replication attempts, no evidence of declining effects appeared. No originating lab's self-confirmatory test ES was significantly different from its self-replication attempt (Fig 2). On average, self-replications were the same size as the self-confirmatory tests $\left(\underline{d}_{\text {difference }}=-0.002, p=.864,95 \% \mathrm{CI}=-0.03-0.03\right.$, between-study heterogeneity $\hat{\tau}=0$ ). In three cases, however, there was a statistically significant difference between the ES in the self-confirmatory test and the average ES across independent replications. As both positive and negative discrepancies occurred, independent replication ESs were the same size as self-confirmatory tests, on average $(\underline{d} \underline{d i f f e r e n c e}=-0.006, p=.838,95 \% \mathrm{CI}=-$ 0.06-0.05). The discrepancies were heterogeneous across studies, with an estimated betweenstudy SD of $\hat{\tau}=0.09$ (95\% CI=0.06-0.16). Based on this degree of heterogeneity, independent replications of new discovery would be predicted to differ from the ES of the self-confirmatory test by as much as $0.2 \mathrm{SD}(95 \%$ prediction interval=-0.2-.21). 

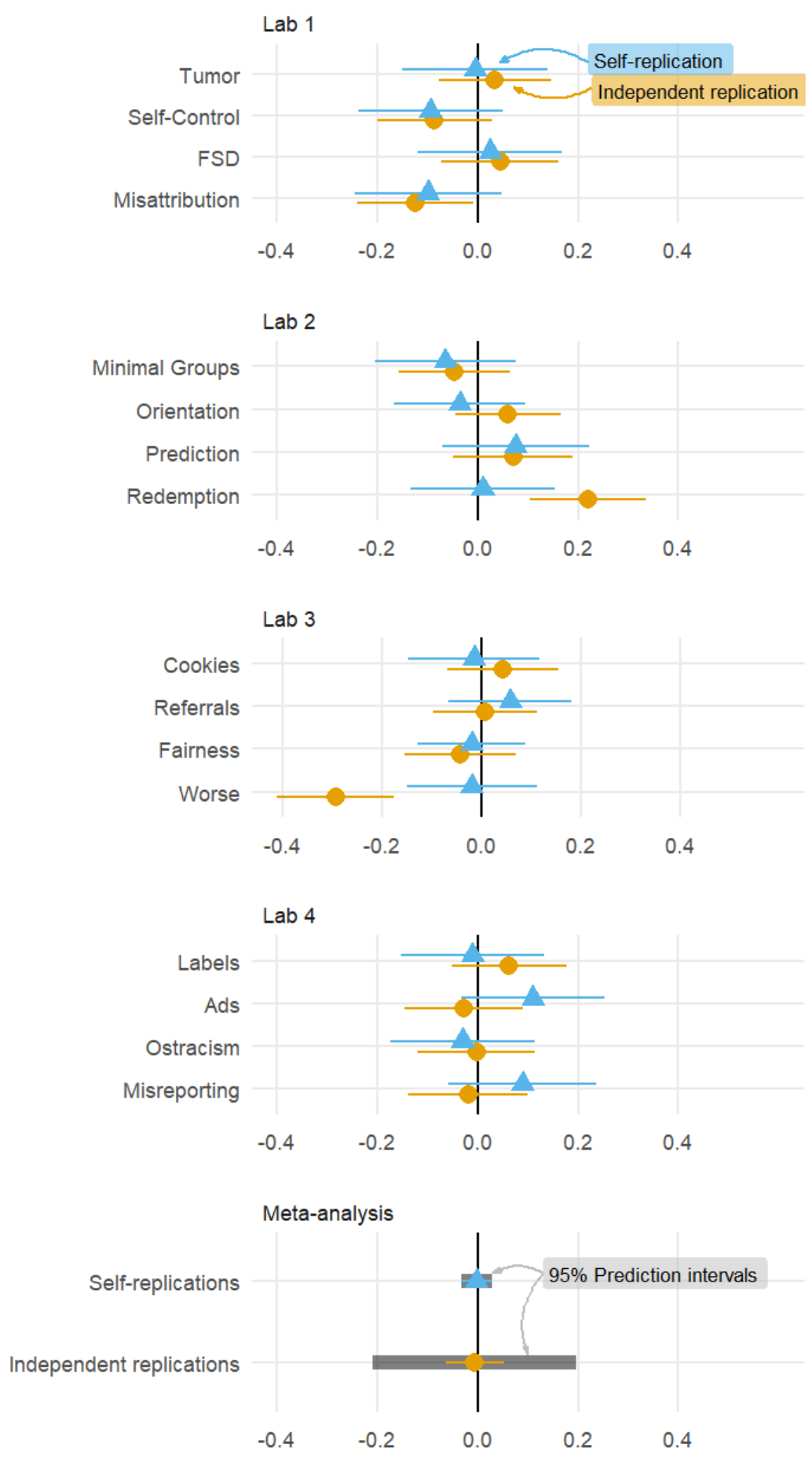

Replication ES - Confirmation ES

Fig 2. Difference in effect sizes (ES) between self- and independent replications for the 16 discoveries, compared with the self-confirmatory test effect size ( 0.0 on the $\mathrm{x}$-axis). Independent replication estimates are fixed-effect meta-analytic estimates of the three independent studies. Meta-analytic estimates with prediction intervals in the bottom panel combine across all 16 discoveries. 
The results of half of the self-confirmatory tests and replications were blinded from data analysis until all replications were completed, to test whether awareness of outcomes influenced replication success. Whether the studies' results were blinded did not moderate the results $(b=-$ $0.01, p=.783$ for differences between self-confirmatory tests and self-replications; $b=0.07$, $p=.206$ for differences between self-confirmatory tests and independent replications). Finally, when testing ESs sequentially over time, no evidence for a decline in ESs from the selfconfirmatory test through final replication was observed $(b=-0.002, p=.701,95 \% \mathrm{CI}=-0.015-$ 0.010; Fig 3). These results did not significantly change when removing the fixed effect for each lab (see Supplementary Information (SI)). In addition, the null was not rejected when comparing the slope of change in ESs among 'blind' vs. 'not blind' studies $(b=0.02, p=.104)$. 


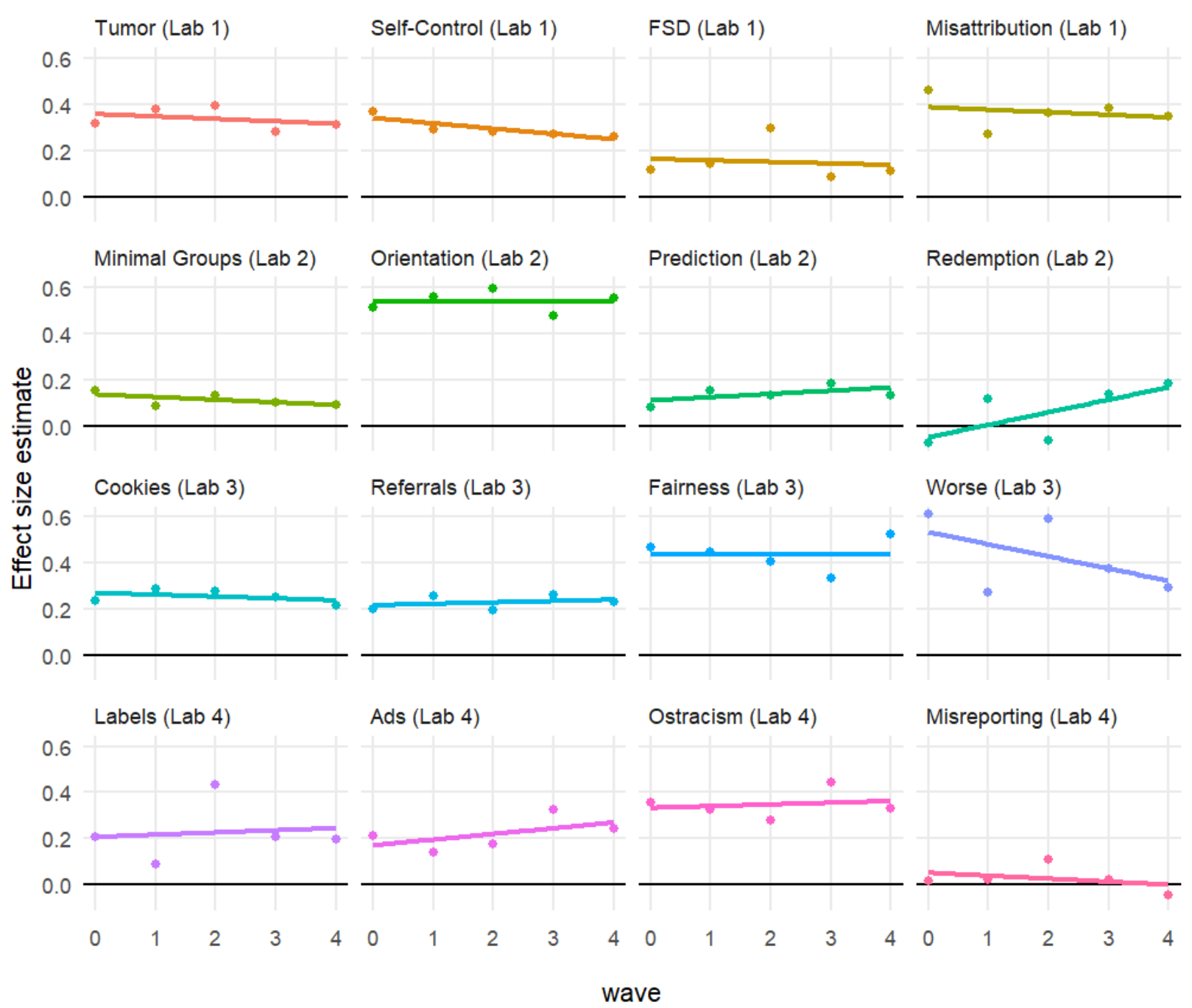

Fig 3: Slope of effect size changes across replications, with the self-confirmatory test as the intercept.

\section{Discussion}

This investigation is unique in exploring replicability. Rather than beginning with published findings and attempting to replicate them in a retrospective replication investigation, we implemented a prospective investigation ${ }^{32,44}$. By discovering new experimental effects, we were able to follow the entire process from discovery to a confirmatory test, and through a sequence of replication attempts. By subjecting ostensible discoveries to large sample, preregistered confirmatory tests $(13 / 16 ; 81 \%$ supported at $p<.05 ; \underline{d}=0.265)$, the primary findings 
for replication were free from $p$-hacking or questionable research practices, unlikely to be artifacts of low statistical power, and fully documented..

These 16 discoveries, four from each of four independent labs, were then subjected to independent —sequential replication $(55 / 64 ; 86 \% p<.05 ; d=0.26)$. Considering replications of only the 13 statistically-significant self-confirmatory tests, the observed replicability rate was 90\%. Considering the power to detect ESs from all 16 self-confirmatory tests, the replication rate could not be expected to be any higher. The replication ESs were the same size on average as self-confirmatory tests when conducted by the discovering labs, 97\% the ES by independent labs. Prior replication efforts in the social-behavioral sciences report replication success rates of about $50 \%$ on average, producing ESs less than half of the originally reported $\mathrm{ES}^{3-13,28}$. The present findings establish a benchmark that high replicability in the social-behavioral sciences is achievable in both statistical inference and ES estimation.

We investigated whether low replicability and declining ESs were an inevitable feature of social-behavioral science - instead, we found a high replicability rate. The present results are reassuring about the effectiveness of what we think of as best practices in scientific investigations. When novel findings were transparently subjected to preregistered, large-sample confirmatory tests — and when replications involved similar materials and were implemented with a commitment to faithfulness to testing the same hypothesis with fidelity to the original procedure - the observed rate of replication was high. Furthermore, we saw no statisticallysignificant evidence of declining effect sizes over replications, either when holding materials, procedures, and sample source constant (except for sampling error) or when materials and procedures and sample sources varied but were faithful to the original studies. 
An uninteresting reason for high replicability would be if the discoveries, although novel, are obviously true. Trivial findings might be particularly easy to replicate. To assess this, we conducted two additional studies (see SI for additional details). In the first, 72 researchers reviewed a synopsis of most of the research designs and predicted the direction of each finding. On average, raters correctly predicted the direction and significance of the self-confirmatory tests $42 \%$ of the time, incorrectly predicted null results $38 \%$ of the time, and incorrectly predicted the direction of the findings $20 \%$ of the time. In the second, 1,180 participants reviewed synopses of the research designs from this study that showed high replicability and from a prior study of published findings from the same fields with similar methodologies that showed low replicability. The synopses were generated by independent researchers with experience in this design. On multiple preregistered criteria, participants were no better at predicting the outcomes of the highly replicable discoveries presented here $\left(M_{\text {present }}\right.$ studies $=40.7 \%$ correct prediction $)$ than at predicting the other less replicable findings from the prior investigation ( $M_{\text {comparison_studies }}=41.1 \%, \hat{\Delta}=-1.65, p<.001$ for a pre-registered equivalence test of $\mathrm{H}_{0}: \Delta \geq 5.0$ ). Notably, the average accuracy rate of researchers in the first study was nearly identical to the average accuracy among lay people in the second. Additionally, the accuracy of predictions for specific findings was significantly associated with the absolute magnitude of the average ESs from independent replications $(b=2.79, \mathrm{z}=2.95, p=-.003$ for the findings in the present study; $b=0.66, \mathrm{z}=3.05, p=.002$ for the comparison findings); absolute ES explained $35 \%$ of the variance in predictability rates. These findings indicate the sample of discoveries used here were not biased towards yielding high replication rates and were neither more obvious or predictable than similar findings with low replication rates. 
It is likely that we observed high replicability because of the rigor-enhancing methodological standards adopted in both the original research leading to discovery and the rigor in replication. First, rather than using exploratory discoveries as the basis for claiming a finding, all discoveries were subjected to preregistered self-confirmatory tests. This eliminated inflation of false positives and ESs by pre-commitment to research designs and analysis plans ${ }^{29}$. Second, once a discovery was submitted for a self-confirmatory test, we committed to reporting the outcomes. This eliminated publication bias that is particularly pernicious when selective reporting of study findings systematically ignores null results ${ }^{30-31}$. Third, all self-confirmatory tests and replications were conducted with large sample sizes (Ns $\gtrsim 1500)$, resulting in relatively precise estimates. Fourth, each lab was part of the process of both discovering and replicating findings. This may have motivated teams to be especially careful in both characterizing their methods and in carrying out their replications. Fifth, if there were essential specialized materials for the experimental design, the discovering lab made them available as supplementary materials. Sharing original materials should increase understanding and adherence to critical features of original experimental methodologies. We expect that all of these features contributed to improving replicability to varying degrees. Future investigations could manipulate these features to learn more about their causal contribution to replicability.

Even when using rigor-enhancing processes, independent replications conducted by other laboratories produced effect estimates that differed from self-confirmatory effect estimates by larger margins than would be expected due to sampling error alone; producing both greater and weaker effect sizes with a heterogeneity on the order of $0.2 \mathrm{SD}$. The added heterogeneity may be due to ambiguities or imprecise descriptions in the materials provided to replicating labs, or due 
to sample heterogeneities introduced by using varying sample providers. This finding highlights the value of multi-lab replication processes even when original studies follow rigorous practices.

An important question is the constraints on the generalizability of these findings to other research. Our 16 novel findings in social-behavioral sciences each involved two between-subject conditions that could be administered online. All samples for self-confirmatory tests and replications were drawn from online panels of American adults. These 16 findings do not characterize a representative sample of any methodology or discipline, although they do represent common methodologies, samples, and research questions from the social-behavioral sciences. Due to the limited number of participating labs, modeling lab-level variation in replicability of findings was incalculable; to the extent that labs vary in how they select potential replication targets, replication rates observed in the present study may not generalize to a broader population of research groups, albeit-as discussed above-the discovered effects did not differ from similar findings when asking laypeople and a group of researchers to predict them.

The most obvious areas for further testing of the replicability ceiling include: more complex experimental or observational designs, in-person behavioral designs that have intricate staging or unusual protocols, sampling strategies that are more inclusive of the world's population, more heterogeneous conditions including variations in procedure and time between investigations, and research domains beyond the social-behavioral sciences. It is possible that our computer-administered protocols are "inherently" more replicable, though prior replication efforts of similar methodologies suggest that this is not the case ${ }^{8-9,12}$, and our follow-up investigation found that the present findings were no more predictable a priori than other findings with similar methodologies that frequently failed to replicate (see SI). Systematic 
investigation will be fruitful for understanding the boundary conditions for achieving high replicability.

Low replicability or declining ESs in social-behavioral research are not inevitable. We did not observe declining effects due to idiosyncrasies of different laboratory practices or different sampling conditions. Whereas prior research demonstrates such declines can occur, the present research demonstrates they do not necessarily occur. The encouraging evidence here should empower scientists with confidence that what we believe to be rigor-enhancing practices may indeed efficiently generate reliable insights. With the adoption of rigorous research practices such as confirmatory testing, large samples, preregistration, strong documentation, and fidelity of replication, high replicability is achievable — perhaps even likely.

\section{Methods}

The four participating labs conducted pilot and exploratory research in the social sciences pursuing their own typical practices and research interests independently of the other labs. Labs were encouraged to investigate any aspect of social-behavioral science, with the requirements that the discoveries submitted for self-confirmatory test and replication be novel, non-obvious, and not involve deception.

Labs submitted promising discoveries for self-confirmatory tests and replication if they met inclusion criteria: a two-group between-subjects manipulation with one focal dependent variable, methods administered via computer online to adults within a single 20-minute study session, and human subjects approval obtained from an Institutional Review Board.

Prior to conducting the self-confirmatory test, the discovering labs preregistered the study design and analysis plan, including materials, protocol, plans for data cleaning and exclusion, 
and specification of the analysis model. Once a self-confirmatory test was preregistered, the lab wrote a methods section to share with the other labs. These methods sections had to include everything that the discovering lab believed would be required for an independent lab to conduct an effective replication. This was done to capture the naturalistic conditions when a researcher reads a methods section and conducts a replication based on it.

Following preregistration, no changes could be made to the methods or procedures, and all labs were committed to replicating the protocol regardless of the outcome of the selfconfirmatory test. The discovering lab conducted its self-confirmatory test with about 1500 participants, and then the project coordinator initiated the replication process with the other labs. Labs were assigned the order to conduct replications in a Latin square design to equate labspecific effects across the order of replications (see SI).

\section{Sharing Study Descriptions}

After a lab identified an ostensible discovery for a self-confirmatory test, they distributed a description of the methodological details that they believed would be required for an independent lab to run a replication. When the replicating labs considered the instructions to be ambiguous on a meaningful part of the design (71\% of studies), replicating labs sought clarifications about methodology from the discovering lab. Usually these were trivial clarifications or confirmations, but not always (see SI).

Replications were done sequentially following the same protocol as self-confirmatory tests, including preregistration. Variation from the 1500 participants per study was due to idiosyncrasies in how the panels and labs managed participant flow and application of 
preregistered exclusion criteria. In most cases, panels allowed more participants to complete the questionnaire.

The discovering labs could specify required exclusion criteria, such as attention checks. Replicating labs could also choose to preregister and implement exclusions for attention checks following their own laboratory's best practices. This was done to capture the natural way researchers conduct replications using their own view of best practices. To maintain ecological validity of labs conducting research in their own style, and to maximize the independence of each replication, all sharing of materials was managed by a project coordinator to prevent unintended communication of designs or results (SI).

\section{Main Studies}

Sixteen new discoveries of social-behavioral phenomena were submitted to selfconfirmatory test and replication, four from each of the participating laboratories. Table 1 catalogs the new discoveries with a brief name, one-sentence summary of the finding, and citation to the research. Table $\mathbf{S 2}$ in the $\mathbf{S I}$ provides links to comprehensive information for each self-confirmatory test and replication, including the preregistration with design and analysis plan, research materials, data, analysis code, analysis output, and written reports of the methods and results.

Table 1: Name, description, and citation or online location of the self-confirmatory tests for the 16 discoveries included in this meta-analysis.

Study Name Description of Central Result $\quad$ Citation

\begin{tabular}{lll}
\hline Tumor & $\begin{array}{l}\text { People hold others responsible for their past good behavior caused } \\
\text { entirely by a brain tumor but not responsible for their past bad } \\
\text { behavior. }\end{array}$ & https://osf.io/4n8pf/ \\
Minimal & People demonstrate less ingroup favoritism when they've been & https://osf.io/adrbe/
\end{tabular}


Groups changed from one minimal group to another compared to when they have not changed group membership.

People will be seen as greedier when they take three of the same Cookies $\quad$ kind of (free) cookie than when they take three different (free) cookies.

When a researcher uses a label to describe people who hold a Label

Self-Control

Orientation

Referrals

Ads

FSD

Prediction

Fairness

Ostracism

Misattribution certain opinion, he or she is interpreted as disagreeing with those attributes when a negative label is used and agreeing with those attributes when a positive label is used.

Someone who commits battery due to self-control failure is less likely to be found guilty if their failure was due to brain damage instead of genes for low self-control.

People judge same-sex interactions as more indicative of the sexuality of men compared to that of women.

People think it is less appropriate to send product referrals when they are the sender of the referral than when they are merely judging the behavior of someone else who sends the referral.

Watching a short ad within a soap-opera episode increases one's likelihood to recommend and promote the company in the ad.

Forcing people to answer questions quickly makes them give more socially desirable answers.

People make more complicated sets of predictions when asked to do so without having the opportunity to explore data.

People evaluate the fairness of punishments that can be expressed in multiple currencies (e.g., time and money) based on whichever currency is initially more salient, but update their fairness judgments when the translation to the other currency is highlighted.

People who are ostracized by computers in an online ball-tossing game become less trusting toward people in general.

People misattribute the feeling of a-ha! they feel while solving an anagram to the truth of the statement the anagram is embedded in. https://osf.io/3vz4k/

?view_only=da108

96b68fe4420bf6c65

a3a7bd64f6

https://osf.io/xq5jb/

?view only $=5305 \mathrm{a}$

812208d48bba3e54

$\underline{6 b f e 38 c 6 c 24}$

Ref. 33

$\underline{\text { https://osf.io/s6qdv/ }}$

https://osf.io/v3thd/

?view_only $=1 \mathrm{c} 328$

08a01ee4c8c81648

0825ad5bebf

https://osf.io/ngz5k/

?view only=8cf18a

2babc1499e98ef57

dbb9926a80

Ref. 34

https://osf.io/e2sf8/

Ref. 35

https://osf.io/58vz9/

?view_only $=312 \mathrm{~b} 6$

$\underline{136155849 \mathrm{a} 79 \mathrm{f} 341}$

$\underline{6933 a 05789 b}$

Ref. 36 
People who look different after committing a transgression seem

Redemption

Misreporting more remorseful, trustworthy, and deserving of a second chance than people who look the same as they did at the time of their transgression.

People evaluate Team B more favorably when they are told Worse $\quad$ Team A is more qualified than Team B than when they are told Team B is less qualified than Team A.

People will report not engaging in an activity if they learn that reporting they have engaged in the activity will result in several additional questions. http://osf.io/6h5s2/

Ref. 37

https://osf.io/3ud4s/

?view_only=420da

$\underline{5 b 8113 b 42 b e 8710 c}$ d7c4b4af14a

\section{Participants}

The population of interest for the self-confirmatory tests and replications was adults living in the United States who could read and write in English. Participants were members of panels that had been recruited through non-probability sampling methods to complete online questionnaires in return for small amounts of money or redeemable 'points' ${ }^{39}$. Labs contracted with different sample providers to provide participants (Stanford: Toluna, SSI, and Dynata; UCSB: CriticalMix, Berkeley: Luth; University of Virginia: SoapBox Sample, Lightspeed GMI). We used different sample providers to minimize potential overlap in sampling, although we cannot be sure that some participants are not part of multiple panels and also repeated our studies as part of different panels. These samples were taken from the providers' online, opt-in, nonprobability panels. The sample providers were instructed to provide American adults drawn in a stratified way with unequal probabilities of selection from the panels so that the people who completed each survey would resemble the nation's adult population (according to the most recently available Current Population Survey, conducted by the U.S. Census Bureau) in terms of gender, age, education, ethnicity (Hispanic vs. not), race (allowing each respondent to select more than one race), region, and income. This method produced samples designed to look similar to probability samples on the matched characteristics but may still have differed in 
unknown ways on unmatched characteristics. Sample providers may have varied in their success at achieving representativeness. A potential lack of adherence to that sampling plan was nonconsequential for the conducted studies. For none of the discoveries were the findings presumed to be limited to a subsample of adults, although there may have been a priori or post facto hypothesizing about moderation by demographic variables. For pilot and exploratory studies, labs used whatever samples they wished (e.g., panel, mTurk, participants visiting the laboratory).

\section{Blinding and sample splitting manipulations}

Two planned manipulations of secondary interest were included to explore potential reasons for variation in the replicability rate or its decline over time. One involved randomly assigning participant recruitment for each data collection of 1500 participants into a first and second wave of 750 to investigate declines in effect size across a single data collection. We assign less confidence to this manipulation, however, as not all panels may have consistently followed our strict protocols for this random assignment (see SI).

For the second manipulation, in 8 of the 16 new discoveries ( 2 from each team), all team members were blind to results of the primary outcome variable from the self-confirmatory tests and replications until all replications for that finding had been completed. For the other 8 discoveries, the data were analyzed and reported to the other teams as the results became available. This was to determine whether explicitly blinding research findings would moderate replicability rates.

\section{Confirmatory Analysis}

\section{Meta-analysis}


In all analyses, meta-analytic models estimated with restricted maximum likelihood were used, as implemented in the 'metafor' package for $\mathrm{R}^{39}$. For single-level models, Knapp-Hartung corrections for standard errors were used. For multilevel models, cluster-robust variance estimation with small-sample corrections were used to account for the limited number of independent studies ${ }^{39}$. Preregistration of the overall analysis plan is available at https://osf.io/6t9vm.

We summarized the overall distribution of effects using a multilevel meta-analysis model, including fixed effects to distinguish replications from self-confirmatory tests, with random effects for each unique discovery and each unique effect size nested within discovery ${ }^{40}$. The study-level variance component describes heterogeneity in the phenomena investigated in different studies and labs. The effect size-level variance component describes heterogeneity across replications of the same phenomena.

\section{Confirmation vs. Self-Replication and Independent Replications}

A random-effects meta-analysis was estimated to analyze the differences between the self-confirmatory test and the replication of the same discovery by the same lab. A negative average change would be evidence of declining replication effect size, even when conducted by the same investigators.

Comparing self-confirmatory tests to replication results from other labs allows for assessment of the impact of between-lab differences in replicability success. Again a randomeffects meta-analysis was used to analyze differences between the self-confirmatory test and the average effect size estimate in the three independent replications. Negative average differences would be evidence of declining replication effect sizes in cross-lab replication. The random- 
effects model provides an estimate of heterogeneity in the differences between self-confirmatory tests and replications beyond what would be expected by sampling error alone. Positive heterogeneity would indicate that effect sizes from self-confirmatory tests could not be exactly replicated by independent labs.

\section{Slope Across Replications}

According to one theory, declines in effect sizes over time are caused by a study being

repeatedly run $^{25}$. If accurate, the more studies run between the self-confirmatory test and the selfreplication, then the greater the decline. To examine temporal decline effects across all replications, we aggregated effect size estimates from each self-confirmatory test with each of the replications and conducted a meta-analytic growth curve. The model also included random effects for each self-confirmatory test or replication attempt of each study that were allowed to covary within study according to an auto-regressive structure. Effect sizes were re-coded for this analysis so that all effects were positive and a slope to non-significance or weakening effect size would be negative in sign.

\section{Data Availability}

Data for each of the individual studies can be found following the OSF links presented in Table S1 in the SI. Data for the overall analysis presented here, along with statistical code, can be found at https://osf.io/bnq5j/. 


\section{References}

1. Putnam, H. (1975). Mathematics, Matter and Method: Volume 1, Philosophical Papers. CUP Archive.

2. Schmidt, S. (2009). Shall we really do it again? The powerful concept of replication is neglected in the social sciences. Review of General Psychology, 13(2), 90-100. https://doi.org/10.1037/a0015108

3. Begley, C. G., \& Ellis, L. M. (2012). Drug development: Raise standards for preclinical cancer research. Nature, 483(7391), 531. https://doi.org/10.1038/483531a

4. Camerer, C. F., Dreber, A., Forsell, E., Ho, T. H., Huber, J., Johannesson, M., et al. (2016). Evaluating replicability of laboratory experiments in economics. Science, 351(6280), 1433-1436. https://doi.org/10.1126/science.aaf0918

5. Camerer, C. F., Dreber, A., Holzmeister, F., Ho, T. H., Huber, J., Johannesson, M., ... \& Altmejd, A. (2018). Evaluating the replicability of social science experiments in Nature and Science between 2010 and 2015. Nature Human Behaviour, 2(9), 637. https://doi.org/10.1038/s41562-018-0399-z

6. Open Science Collaboration. (2015). Estimating the reproducibility of psychological science. Science, 349(6251), aac4716. https://doi.org/10.1126/science.aac4716

7. Errington, T. M., Mathur, M., Soderberg, C. K., Denis, A., Perfito, N., Iorns, E., \& Nosek, B. A. (2021). Investigating the replicability of preclinical cancer biology. Elife, 10, e71601. https://doi.org/10.7554/eLife.71601

8. Klein, R. A., Vianello, M., Hasselman, F., Adams, B. G., Adams Jr, R. B., Alper, S., ... \& Batra, R. (2018). Many Labs 2: Investigating variation in replicability across samples and settings. Advances in Methods and Practices in Psychological Science, 1(4), 443-490. https://doi.org/10.1177/2515245918810225

9. Ebersole, C. R., Atherton, O. E., Belanger, A. L., Skulborstad, H. M., Allen, J. M., Banks, J. B.,... \& Brown, E. R. (2016). Many Labs 3: Evaluating participant pool quality across the academic semester via replication. Journal of Experimental Social Psychology, 67, 68-82. https://doi.org/10.1016/j.jesp.2015.10.012

10. Wagenmakers, E. J., Beek, T., Dijkhoff, L., Gronau, Q. F., Acosta, A., Adams Jr, R. B., ... \& Bulnes, L. C. (2016). Registered replication report: Strack, Martin, \& Stepper (1988).

Perspectives on Psychological Science, 11(6), 917-928. https://doi.org/10.1177/1745691616674458

11. O’Donnell, M., Nelson, L. D., Ackermann, E., Aczel, B., Akhtar, A., Aldrovandi, S., ... \& Balatekin, N. (2018). Registered replication report: Dijksterhuis and van Knippenberg (1998). Perspectives on Psychological Science, 13(2), 268-294. https://doi.org/10.1177/1745691618755704 
12. Piovesan, M., Døssing, F. S., \& Wengström, E. R. (2017). Registered Replication Report: Rand, Greene \& Nowak (2012). Perspectives on Psychological Science. 12(3) 527-542. http://doi.org/10.1177/1745691617693624

13. Verschuere, B., Meijer, E. H., Jim, A., Hoogesteyn, K., Orthey, R., McCarthy, R. J., ... \& Barbosa, F. (2018). Registered replication report on Mazar, Amir, and Ariely (2008). Advances in Methods and Practices in Psychological Science, 1(3), 299-317.

https://doi.org/10.1177/2515245918781032

14. Gilbert, D. T., King, G., Pettigrew, S., \& Wilson, T. D. (2016a). Comment on "Estimating the reproducibility of psychological science. Science, 351(6277).

https://doi.org/10.1126/science.aad7243

15. Wilson, B. M., Harris, C. R., \& Wixted, J. T. (2020). Science is not a signal detection problem. Proceedings of the National Academy of Sciences, 117(11), 5559-5567.

https://doi.org/10.1073/pnas.1914237117

16. Reich, E. S. (2012). Timing glitches dog neutrino claim. Nature News, 483(7387), 17. https://doi.org/10.1038/483017a

17. Simmons, J. P., Nelson, L. D., \& Simonsohn, U. (2011). False-positive psychology: Undisclosed flexibility in data collection and analysis allows presenting anything as significant. Psychological science, 22(11), 1359-1366.

https://doi.org/10.1177/0956797611417632

18. Van Bavel, J. J., Mende-Siedlecki, P., Brady, W. J., \& Reinero, D. A. (2016). Contextual sensitivity in scientific reproducibility. Proceedings of the National Academy of Sciences, 113(23), 6454-6459. https://doi.org/10.1073/pnas.1521897113

19. Hedges, L. V., \& Schauer, J. M. (2019). Consistency of effects is important in replication: Rejoinder to Mathur and VanderWeele (2019). Psychological Methods, 24(5), https://doi.org/10.1037/met0000237

20. Glymour, C., Scheines, R., \& Spirtes, P. (2014). Discovering causal structure: Artificial intelligence, philosophy of science, and statistical modeling. Academic Press.

21. Protzko, J. (Under Review) Null-hacking, a lurking problem. https://psyarxiv.com/9y3mp/

22. Bryan, C., Yeager, D. S., \& O’Brien, J. (2019). Replicator Degrees of Freedom Allow Publication of Misleading 'Failures to Replicate'. Available at SSRN 3408200. https://doi.org/10.2139/ssrn.3408200

23. Nosek, B. A., \& Errington, T. M. (2020). What is Replication? PLOS Biology. https://doi.org/10.1371/journal.pbio.3000691 
24. Schooler, J. (2011). Unpublished results hide the decline effect: some effects diminish when tests are repeated. Jonathan Schooler says being open about findings that don't make the scientific record could reveal why. Nature, 470(7335), 437-438. https://doi.org/10.1038/470437a

25. Protzko, J., \& Schooler, J. W. (2017). Decline effects: Types, mechanisms, and personal reflections. Psychological science under scrutiny: Recent challenges and proposed solutions, 85107. https://doi.org/10.18637/jss.v036.i03

26. Yeager, D. S., Krosnick, J. A., Visser, P. S., Holbrook, A. L., \& Tahk, A. M. (2019). Moderation of classic social psychological effects by demographics in the US adult population: New opportunities for theoretical advancement. Journal of Personality and Social Psychology. 117(6), e84-e99. http://dx.doi.org/10.1037/pspa0000171

27. Richard, F. D., Bond Jr, C. F., \& Stokes-Zoota, J. J. (2003). One hundred years of social psychology quantitatively described. Review of General Psychology, 7(4), 331-363. https://doi.org/10.1037/1089-2680.7.4.331

28. Kvarven, A., Strømland, E., \& Johannesson, M. (2019). Comparing meta-analyses and preregistered multiple-laboratory replication projects. Nature Human Behaviour, 1-12. https://doi.org/10.31219/osf.io/brzwt

29. Wagenmakers, E. J., Wetzels, R., Borsboom, D., van der Maas, H. L., \& Kievit, R. A. (2012). An agenda for purely confirmatory research. Perspectives on Psychological Science, 7(6), 632-638. https://doi.org/10.1177/1745691612463078

30. Greenwald, A. G. (1975). Consequences of prejudice against the null hypothesis. Psychological Bulletin, 82, 1-20. https://doi.org/10.1037/h0076157

31. Rosenthal, R. (1979). The file drawer problem and tolerance for null results. Psychological Bulletin, 86, 638-641. https://doi.org/10.1037/0033-2909.86.3.638

32. Schweinsberg, M., Madan, N., Vianello, M., Sommer, S. A., Jordan, J., Tierney, W., ... \& Srinivasan, M. (2016). The pipeline project: Pre-publication independent replications of a single laboratory's research pipeline. Journal of Experimental Social Psychology, 66, 55-67.

33. Protzko, J., Buttrick, N., Ebersole, C. R., Lundmark, S., \& Schooler, J. (2020, May 13). Not all Explanations are Equal: Only Explanations Invoking a Change from the true self Mitigate Responsibility. Retrieved from psyarxiv.com/sx7cn. https://doi.org/10.31234/osf.io/sx7cn

34. Protzko, J., Zedelius, C. M., \& Schooler, J. W. (2019). Rushing to Appear Virtuous: Time Pressure Increases Socially Desirable Responding. Psychological science, 30(11), 1584-1591. https://doi.org/10.1177/0956797619867939

35. Evers, E. R. K., O'Donnell, M., \& Inbar, Y. (2019, September 18). Arbitrary Fairness in Rewards and Punishments. https://doi.org/10.31234/osf.io/gnr9f 
36. Laukkonen, R. E., Kaveladze, B. T., Protzko, J., Tangen, J. M., von Hippel, W., \& Schooler, J. W. (2022). Irrelevant insights make worldviews ring true. Scientific Reports, 12(1), 2075.

37. Inbar, Y., \& Evers, E. R. K. (2019, September 12). Worse is Bad: Divergent Inferences From Logically Equivalent Comparisons. https://doi.org/10.31234/osf.io/ueymx

38. Viechtbauer, W. (2010). Conducting meta-analyses in R with the metafor package. Journal of Statistical Software, 36(3), 1-48. URL: http://www.jstatsoft.org/v36/i03/

39. Tipton, E. \& Pustejovsky, J. E. (2015). Small-sample adjustments for tests of moderators and model fit using robust variance estimation in meta-regression. Journal of Educational and Behavioral Statistics, 40(6), 604-634. https://doi.org/10.3102/1076998615606099

39. Konstantopoulos, S. (2011). Fixed effects and variance components estimation in three-level meta-analysis. Research Synthesis Methods, 2(1), 61-76. https://doi.org/10.1002/jrsm.35

40. Iso-Ahola, S. E. (2017). Reproducibility in psychological science: When do psychological phenomena exist?. Frontiers in Psychology, 8, 879.

41. Iso-Ahola, S. E. (2020). Replication and the establishment of scientific truth. Frontiers in psychology, 11, 2183.

42. Strack, F., \& Stroebe, W. (2018). What have we learned? What can we learn. Behavioral and Brain Sciences, 41, e151.

43. Stroebe, W., \& Strack, F. (2014). The alleged crisis and the illusion of exact replication. Perspectives on Psychological Science, 9(1), 59-71.

44. Schooler, J. W. (2014). Metascience could rescue the 'replication crisis'. Nature, 515(7525), 9. https://doi.org/10.1038/515009a 


\section{Acknowledgments}

We thank Bruce Fetzer and Mallory Kidwell for their help with the project. We also thank Brett Ouimette, Pia Noel, Blair Schembari, Madeleine Gross, Benji Kaveladze, Ruben Laukkonen, Natalie Buchen, Rachel Narr, Maura Austin, Jamie Rodriguez, Karen Kurosawa, Hope Harrington, Michael Basili, and Dustin Dienhart for their help in completing the individual studies comprising this project. We thank Suzanne Hoogeveen, Alexandra Sarafoglou, Anna Dreber Almenberg, and EJ Wagenmakers for help creating the study descriptions. This work was sponsored by the Fetzer Franklin Fund of the John E. Fetzer Memorial Trust to each of the participating laboratories at the University of California-Santa Barbara, University of Virginia, University of California-Berkeley, and Stanford University.

\section{Author Contributions}

John Protzko: Conceptualization, Methodology, Validation, Formal Analysis, Investigation, Resources, Data Curation, Writing - Original Draft, Writing - Review \& Editing, Project administration Jon Krosnick: Conceptualization, Methodology, Validation, Investigation, Data Curation, Writing - Review \& Editing, Supervision, Funding acquisition Leif Nelson: Conceptualization, Validation, Investigation, Resources, Writing - Review \& Editing, Supervision, Funding acquisition Brian A. Nosek: Conceptualization, Validation, Investigation, Resources, Writing - Review \& Editing, Visualization, Supervision, Funding acquisition Jordan Axt: Validation, Investigation, Resources, Writing - Review \& Editing Matt Berent: Methodology, Validation, Investigation, Resources, Data Curation, Supervision, Writing Review \& Editing Nick Buttrick: Validation, Investigation, Resources, Writing - Review \& Editing Matthew DeBell: Validation, Formal Analysis, Investigation, Resources, Writing Review \& Editing Charles R. Ebersole: Validation, Investigation, Resources, Writing Reviewing \& Editing Sebastian Lundmark: Software, Validation, Investigation, Resources, Data Curation, Writing - Review \& Editing Bo MacInnis: Validation, Investigation, Resources, Writing Reviewing and Editing Michael O'Donnell: Validation, Investigation, Resources, Writing - Review \& Editing Hannah Perfecto: Validation, Investigation, Resources, Writing Review \& Editing James Pustejovsky: Methodology, Formal Analysis, Data curation, Writing Review \& Editing, Visualization Scott Roeder: Validation, Investigation, Resources Jan Walleczek: Conceptualization, Methodology, Writing - Review \& Editing, Supervision, Project administration, Funding acquisition Jonathan W. Schooler: Conceptualization, Methodology, Investigation, Project Administration, Writing - Original Draft, Writing - Review \& Editing, Supervision, Funding acquisition.

Author names are presented in the following order: Project Manager (JP), Individual PIs in alphabetical order (JK, LN, BAN), then authors in alphabetical order (JA, MB, NB, MD, CRE, SL, BM, MO, HP, JEP, SR, JW), Project PI and senior author (JWS). 


\section{Competing Interests}

B.A.N. is the Executive Director of the nonprofit Center for Open Science with a mission to "increase openness, integrity, and reproducibility of research." J.W.S. was the Scientific Director of the Fetzer Franklin Fund that sponsored this research and B.A.N. was also on the scientific advisory board. J.W.S. made substantive contributions to the design and execution of this research, but as a funder did not have controlling interest in the decision to publish or not. All other authors declare no conflicts of interest. 


\section{Supplementary Information:}

\section{High Replicability of Newly-Discovered Social-behavioral Findings is Achievable}

John Protzko*1,15, Jon Krosnick ${ }^{2}$, Leif Nelson ${ }^{4}$, Brian A. Nosek ${ }^{3,7}$, Jordan Axt ${ }^{5}$, Matt Berent ${ }^{6}$, Nick Buttrick ${ }^{7}$, Matthew DeBell ${ }^{2}$, Charles R. Ebersole ${ }^{7}$, Sebastian Lundmark ${ }^{9}$, Bo MacInnis ${ }^{2}$, Michael O’Donnel1 ${ }^{10}$, Hannah Perfecto ${ }^{11}$, James E. Pustejovsky ${ }^{12}$, Scott Roeder ${ }^{13}$, Jan Walleczek ${ }^{14}$, Jonathan W. Schooler ${ }^{1}$

${ }^{1}=$ University of California, Santa Barbara $;{ }^{2}=$ Stanford University; ${ }^{3}=$ Center for Open Science $;{ }^{4}=$ University of California, Berkeley; ${ }^{5}=$ McGill University; ${ }^{6}=$ Matt Berent Consulting $;{ }^{7}=$ University of Virginia $;{ }^{9}=$ University of Gothenburg, ${ }^{10}=$ Georgetown University; ${ }^{11}=$ Washington University in St. Louis; ${ }^{12}=$ University of Wisconsin - Madison; ${ }^{13}$ $=$ University of South Carolina; ${ }^{14}=$ Fetzer Franklin Fund; ${ }^{15}=$ Central Connecticut State University 


\section{The Four Participating Laboratories}

The prospective replication project originated from a 2012 meeting about the observation of declining effect sizes between original findings and subsequent investigations organized by Jonathan Schooler at UC Santa Barbara and funded by the Fetzer Franklin Fund. The four participating laboratories were UC Santa Barbara (PI: Jonathan Schooler, Department of Psychology), Stanford University (PI: Jon Krosnick, Department of Communication), UC Berkeley (PI: Leif Nelson, Marketing, Haas School of Business), and University of Virginia (PI: Brian Nosek, Department of Psychology). Each laboratory had prior and subsequent experience independent of this project conducting replications of others' work and having their own studies replicated by others-sometimes successfully and sometimes unsuccessfully ${ }^{1-5}$. This includes some members of the present team failing to replicate prior findings from other members of the present team ${ }^{4}$. Thus, the team was composed of replicators and original authors from prior replication efforts that had experienced a diversity of replication failures and successes concordant with the variability in observing replication success across the social-behavioral sciences.

\section{Additional Procedures}

\section{Video Instructions}

For confirmation and replication studies: video instructions were put before each study. These videos were short, produced by each individual lab, and merely welcomed participants to the study. This was done to add greater individuality to the experiments and more closely emulate a laboratory procedure in which an experimenter would greet participants. 


\section{Blinding.}

In the event that substantial decline in effect sizes was observed, we planned to compare findings where the outcomes of prior investigations were known versus unknown. Before data collection began in the confirmation stage, each study was designated as either 'not blind' or 'blind'. Studies that were not blind proceeded as any study would. Studies that were 'blind' were blinded to prevent the lab from interacting with, analyzing, or observing the dependent measure in their study until all other laboratories had collected their replications. Thus, a 'blind' study would be run, the data collected, but the primary DV untouched, not looked at, nor analyzed until all other labs had replicated the work. For any blind study, all replications were also kept 'blind' until data collection was completed for the last replication.

\section{Participants and the 750/750 split}

To facilitate examination of unusual reasons for declining effects, had they been observed, we planned to make comparisons within each data collection based on the first and second half of the data collected. Because minimal decline was observed, this plan and analyses are not emphasized in the main text. We retain a full description of this feature of the design here for completeness.

All participants from each confirmation and replication were collected using the labs' individual online sample provider, using the demographic criteria. Online sample providers were given the following instructions:

Draw the sample to be sufficiently large to include more than enough participants to achieve 1500 completed studies (and passing attention/quality control checks) within two weeks of inviting all of those people to complete the survey. River sampling and 
routers were not allowed to be used to obtain participants, as they do not allow selection of the entire potential sample and randomly splitting it in half before data collection begins.

Furthermore, data collections involving a confirmation or replication phase study were collected in two immediate waves of 750 respondents instead of collecting all 1500 participants simultaneously. Hence, after the full sample had been drawn, but before participants were invited to take the study, the drawn sample was divided into two truly random halves. This random assignment was done using random numbers obtained from the Random.org random integer generator (https://www.random.org/integers/?mode=advanced/).

Specifically, the online sample providers were instructed to download random numbers from the generator in batches of 10,000, with each integer having a random value between 1 and 10,000, using 1 column, decimal numeral system, and having "Generate your own personal randomization right now" checked. One lab drew their own numbers and supplied them to the sample provider; the other three labs instructed their online sample provider to draw the numbers themselves. Each number drawn was appended to one participant in the full sample, until all participants had been assigned one number each. Participants who were assigned even random numbers were treated as belonging to the sample that was first invited to complete the questionnaire and people who were assigned odd random numbers were treated as belonging to the second sample.

Participants in the first sample were sorted in an ascending order according to the random.org number assigned to each person. Participants in the second sample were sorted in an ascending order according to the random.org number assigned to each 
person. Beginning with the first person in the sorted list of first sample participants, enough participants were invited so that 750 completed studies, with participants passing the attention/quality control check(s), was finished collecting within two weeks of the first invitation sent.

After 750 participants from the first sample completed the questionnaire and passed the attention/quality control check(s), the second sample was invited using the same procedure to yield 750 completed interviews passing the attention/quality control $\operatorname{check}(\mathrm{s})$ by the end of the 14th day after the data collection began. None of the participants in the second sample were allowed to be invited before the first sample had finished collecting and been closed for further collection.

\section{Order of Replications}

Labs were assigned the order to conduct replications in a Latin square design to equate lab-specific effects across order of replications (see Table S1).

Table S1: Order of data collection, analysis, and blinding status. Yellow Highlighting corresponds to replications whose results were blinded until the completion of all studies from that cycle. The results of non-highlighted replications were analyzed and reported to the rest of the team as soon as they were completed. The $1^{\text {st }}$ or $2^{\text {nd }} 750$ refers to which 750 was analyzed first. Bold corresponds to self-replication.

\begin{tabular}{|l|l|l|l|l|l|}
\hline Wave 1 & $\begin{array}{l}\text { Confirmatio } \\
\mathbf{n}\end{array}$ & $\begin{array}{l}\text { Replication } \\
\mathbf{1}\end{array}$ & $\begin{array}{l}\text { Replication } \\
\mathbf{2}\end{array}$ & $\begin{array}{l}\text { Replication } \\
\mathbf{3}\end{array}$ & $\begin{array}{l}\text { Replicatio } \\
\mathbf{n} \mathbf{4}\end{array}$ \\
\hline Lab 1 & Lab 1 & Lab 4 & Lab 2 & Lab 3 & Lab 1 \\
& $1^{\text {st }} 750$ & $1^{\text {st }} 750$ & $2^{\text {nd }} 750$ & $2^{\text {nd }} 750$ & $1^{\text {st }} 750$ \\
Lab 2 & Lab 2 & Lab 3 & Lab 4 & Lab 1 & Lab 2 \\
& $2^{\text {nd } 750}$ & $1^{\text {st }} 750$ & $2^{\text {nd }} 750$ & $2^{\text {nd } 750}$ & $1^{\text {st } 750}$ \\
Lab 3 & Lab 3 & Lab 2 & Lab 1 & Lab 4 & Lab 3 \\
& $1^{\text {st } 750}$ & $2^{\text {nd }} 750$ & $1^{\text {st }} 750$ & $1^{\text {st } 750}$ & $2^{\text {nd } 750}$
\end{tabular}




\begin{tabular}{|c|c|c|c|c|c|}
\hline \multirow{2}{*}{ Lab 4} & Lab 4 & Lab 1 & Lab 3 & Lab 2 & Lab 4 \\
\hline & $2^{\text {nd }} 750$ & $2^{\text {nd }} 750$ & $1^{\text {st }} 750$ & $1^{\text {st }} 750$ & $2^{\text {nd }} 750$ \\
\hline Wave 2 & $\begin{array}{l}\text { Confirmatio } \\
\mathrm{n}\end{array}$ & $\begin{array}{l}\text { Replication } \\
1\end{array}$ & $\begin{array}{l}\text { Replication } \\
2\end{array}$ & $\begin{array}{l}\text { Replication } \\
3\end{array}$ & $\begin{array}{l}\text { Replicatio } \\
\text { n } 4\end{array}$ \\
\hline \multirow{2}{*}{ Lab 1} & Lab 1 & Lab 3 & Lab 4 & Lab 1 & Lab 2 \\
\hline & $2^{\text {nd }} 750$ & $2^{\text {nd }} 750$ & $2^{\text {nd }} 750$ & $1^{\text {st }} 750$ & $1^{\text {st }} 750$ \\
\hline \multirow{2}{*}{ Lab 2} & Lab 2 & Lab 1 & Lab 3 & Lab 2 & Lab 4 \\
\hline & $1^{\text {st }} 750$ & $1^{\text {st }} 750$ & $1^{\text {st }} 750$ & $2^{\text {nd }} 750$ & $2^{\text {nd }} 750$ \\
\hline & Lab 3 & Lab 4 & Lab 2 & Lab 3 & Lab 1 \\
\hline Lab 3 & $2^{\text {nd }} 750$ & $1^{\text {st }} 750$ & $1^{\text {st }} 750$ & $2^{\text {nd }} 750$ & $2^{\text {nd }} 750$ \\
\hline \multirow{2}{*}{ Lab 4} & Lab 4 & Lab 2 & Lab 1 & Lab 4 & Lab 3 \\
\hline & $1^{\text {st }} 750$ & $2^{\text {nd }} 750$ & $2^{\text {nd }} 750$ & $1^{\text {st }} 750$ & $1^{\text {st }} 750$ \\
\hline Wave 3 & $\begin{array}{l}\text { Confirmatio } \\
\text { n }\end{array}$ & $\begin{array}{l}\text { Replication } \\
1\end{array}$ & $\begin{array}{l}\text { Replication } \\
2\end{array}$ & $\begin{array}{l}\text { Replication } \\
3\end{array}$ & $\begin{array}{l}\text { Replicatio } \\
\text { n } 4\end{array}$ \\
\hline \multirow{2}{*}{ Lab 1} & Lab 1 & Lab 1 & Lab 3 & Lab 2 & Lab 4 \\
\hline & $1^{\text {st }} 750$ & $2^{\text {nd }} 750$ & $1^{\text {st }} 750$ & $1^{\text {st }} 750$ & $2^{\text {nd }} 750$ \\
\hline \multirow{3}{*}{ Lab 2} & Lab 2 & Lab 2 & Lab 1 & Lab 4 & Lab 3 \\
\hline & $2^{\text {nd }} 750$ & $1^{\text {st }} 750$ & $2^{\text {nd }} 750$ & $2^{\text {nd }} 750$ & $1^{\text {st }} 750$ \\
\hline & Lab 3 & Lab 3 & Lab 4 & Lab 1 & Lab 2 \\
\hline Lab 3 & $1^{\text {st }} 750$ & $2^{\text {nd }} 750$ & $1^{\text {st }} 750$ & $1^{\text {st }} 750$ & $2^{\text {nd }} 750$ \\
\hline \multirow{2}{*}{ Lab 4} & Lab 4 & Lab 4 & Lab 2 & Lab 3 & Lab 1 \\
\hline & $2^{\text {nd }} 750$ & $1^{\text {st }} 750$ & $2^{\text {nd }} 750$ & $2^{\text {nd }} 750$ & $1^{\text {st }} 750$ \\
\hline Wave 4 & $\begin{array}{l}\text { Confirmatio } \\
\mathrm{n}\end{array}$ & $\begin{array}{l}\text { Replication } \\
1\end{array}$ & Replication & $\begin{array}{l}\text { Replication } \\
3\end{array}$ & $\begin{array}{l}\text { Replicatio } \\
\text { n } 4\end{array}$ \\
\hline \multirow{2}{*}{ Lab 1} & Lab 1 & Lab 2 & Lab 1 & Lab 4 & Lab 3 \\
\hline & $2^{\text {nd }} 750$ & $1^{\text {st }} 750$ & $1^{\text {st }} 750$ & $2^{\text {nd }} 750$ & $2^{\text {nd }} 750$ \\
\hline
\end{tabular}




\begin{tabular}{llllll} 
Lab 2 & Lab 2 & Lab 4 & Lab 2 & Lab 3 & Lab 1 \\
& $1^{\text {st } 750}$ & $2^{\text {nd } 750}$ & $2^{\text {nd } 750}$ & $1^{\text {st } 750}$ & $1^{\text {st } 750}$ \\
Lab 3 & Lab 3 & Lab 1 & Lab 3 & Lab 2 & Lab 4 \\
& $2^{\text {nd } 750}$ & $1^{\text {st } 750}$ & $1^{\text {st } 750}$ & $2^{\text {nd } 750}$ & $2^{\text {nd } 750}$ \\
Lab 4 & Lab 4 & Lab 3 & Lab 4 & Lab 1 & Lab 2 \\
& $1^{\text {st } 750}$ & $2^{\text {nd } 750}$ & $2^{\text {nd } 750}$ & $1^{\text {st } 750}$ & $1^{\text {st } 750}$ \\
\hline
\end{tabular}

\section{Overview of Design}

\section{Meta-Study Design}

The overall design involved four laboratories, each of which conducted four original confirmatory studies on research topics of interest that came out of discovery-oriented research. Each study involved a two-group, between-subjects manipulation. Multiple outcomes could be assessed, but labs had to designate a single focal outcome. After a lab identified a finding during the discovery phase that was interesting and eligible for inclusion, they conducted a confirmation study using a new sample of participants. After completing the confirmation studies, each original study was then replicated four times, once by each lab, with order of replications assigned using a Latin square design.

\section{Sample Splits}

Each confirmation study and replication study was conducted using a target sample of at least 1500 participants, split into two halves. When inviting participants to take part in each survey, participants were randomly assigned to be invited to be a part of the first 750 half sample or the second 750 half sample. Sampling firms frequently collected more data than necessary to 
meet the 1500 participant target. Some of this was due to how they managed participant invitations and tracking, and some of this was a function of oversampling so that there would still be 1500 participants after applying exclusion rules for failing attention checks.

\section{Data Analysis}

Confirmation studies that were not 'blind' were analyzed, and a results section was distributed to the other labs within one week of data collection finishing. To match this amount of time among 'blind' studies, one week was artificially imposed as a delay after one lab ran their study and the next lab launched their replication.

\section{Blinding}

As part of the a priori design to assess unusual reasons for declining effects, had they been observed, each initial confirmation study and each replication study was assigned to either a) analyze the first half-sample and then the second half-sample or b) analyze the second halfsample and then the first half-sample. Confirmation studies were randomly assigned to order of observation, blocking by lab. Replication studies were randomly assigned to order of observation, blocking by study within labs. If observer effects cause the decline effect, then whichever 750 was analyzed first should yield larger effect sizes than the 750 that was analyzed second.

Confirmation studies and all of their replications were assigned to be either blind or not blind (two per wave, all labs having two blind studies throughout the project). Blinded studies had all data and replications collected before the dependent variable was observed. Thus, there may be an effect of knowing the results of a study or its confirmation that would influence other labs in their data collection, programming, or analysis efforts. If blinded studies showed 
systematically different effect sizes or different changes in effect size over replications, we would have evidence for causal effects of interacting with data on subsequent replication efforts. For confirmation vs. self-replication tests, we predicted larger declines in non-blinded studies than in blinded studies. This was tested by including a binary term for whether the study was blinded $[$ blind $=1$, normal $=0]$ into the meta-regression. For the change in effect size across 750/750 splits, we predicted blind studies would show a flatter slope than the decline seen in non-blind studies. This was tested by including a binary term for whether the study was blinded $[$ blind $=1$, normal $=0]$ into the meta-regression .

\section{Interactions between laboratories}

By default, labs were discouraged from interacting with each other about replication designs and implementation to maximize independence of tests. Originators of each discovery wrote a complete methods section and provided specialized materials (e.g., videos) if any. The project manager received final methods and circulated them on the planned schedule to the replication labs. If replication labs needed to seek clarification from the originating lab to conduct the replication study, the interaction was limited to clarification of the key question and replication labs returned to designing their replication study independently. Table S3 summarizes the interactions that occurred between laboratories.

Table S3: Instances of replicating labs requesting additional information from the originating lab to run a direct replication of the study. Columns indicate which school requested the additional information.

\begin{tabular}{lllll}
\multirow{2}{*}{ Study Name } & UCSB & UVA & Berkeley & Stanford \\
& Replication & Replication & Replication & Replication
\end{tabular}

Tumor

Additional Info

Given: Clarified 'reverse coded' 
Minimal Groups

Cookies

Label

Helped Stanford design materials and ran a pilot for them.

Self-control

Orientation

Additional Info

Requested

Referrals

Additional Info

Requested

Additional Info

Given: Clarified

randomization

Ads

Fast Social

Desirability

(FSD)

Additional Info

Requested

Additional Info

Prediction

requested

Fairness

Additional Info

Ostracism Given: Javascript

help on Safari

Misattribution

Additional info

Additional Info

requested

Given

Redemption .qsf shared

.qsf shared

.qsf shared

Worse

Misreporting

Additional Info

Given

Overall, for 14 of the 48 (29\%) independent replications there was no interaction between the originating lab and the replication lab beyond sharing the methods section and key materials.

\section{Confirmatory Analyses}




\section{Effect Sizes}

All study results were transformed into a Cohen's d effect size metric, as all studies involved two between-subjects conditions. In the majority of cases this involved computing the effect size from the means and standard deviations. In cases where regression was used conditioning on other variables, or outcomes were dichotomous, effect sizes were calculated using alternate formulae to produce an equivalent Cohen's $\mathbf{d}$. This included transforming an unstandardized regression coefficient using $n \mathrm{~s}$ and the SD of the dependent variable for regression outcomes. Binary outcomes were analyzed with a probit regression and the marginal predicted probabilities and standard errors were used to construct the $\mathbf{d}^{6-7}$.

\section{Coding of Replicability Rate}

Studies were coded as 1 if they produced a statistically significant result in the direction expected by theory and 0 if they did not reach statistical significance [no study showed statistically significant effects in the opposite direction].

\section{Lab-specific Variation}

We then tested two hypotheses, pertaining to the originating lab effects and the replication lab effects. First, we tested the hypothesis $a_{1}=a_{2}=a_{3}=a_{4}$ to examine whether average effect sizes of the confirmation studies differ across labs where $a_{i}$ is a fixed effect for each lab. Second, we examined whether average effect sizes vary depending on the lab conducting the replication study by testing the hypothesis $\gamma_{1}=\gamma_{2}=\gamma_{3}=\gamma_{4}$ (where $\gamma_{\mathrm{i}}$ is a fixed effect for each lab). This was done using likelihood ratio tests (i.e., using model-based methods, rather than robust variance estimation) because of their greater power. We also conducted corresponding tests based on robust variance estimation methods (i.e., robust Approximate 
Hotelling's $\mathrm{T}^{2}$ tests) as sensitivity analyses. As a further sensitivity analysis, we re-estimated the model after removing the time variable $(0=$ confirmatory test/intercept, $1-4$ for 1 st, $2 \mathrm{nd}$, $3 \mathrm{rd}$, and 4th replication), the blinding indicator, and their interaction, as well as simplifying the random effects structure to a study-specific intercept; we then repeated the above hypothesis tests under the reduced model.

\section{Confirmation versus Self-Replication}

We calculated differences in standardized effect sizes, pooling effect size estimates across the two half-samples from each replication, subtracting the confirmation effect size from the self-replication effect size. This way, a negative value corresponds to a decline in effect size. This analysis was done using a random effects meta-analysis with robust standard errors on the difference in the self-replication effect size from the confirmation effect size. We included metaregression fixed effects for the number of replications run by other teams in between the confirmation and self-replication.

\section{0/750 splits}

Confirmatory tests and replication studies had 1,500 or more participants each who were themselves assigned to serve as part of either the first or second set of 750 participants. The $750 / 750$ split was included to investigate the stability of effects over time when all other factors are held constant. Companies were instructed to draw from their panels enough participants so that 1500 completed surveys passing attention and/or quality checks could be obtained. These participants were then randomly assigned by the companies to be invited to take each confirmation and replication as part of the $1^{\text {st }}$ or $2^{\text {nd }} 750$ participants. However, based on some 
characteristics of observed recruiting during data collection, we are not confident that all of the panels effectively implemented this design with random assignment.

This analysis looked at the difference in effect size between the set of participants randomly assigned to be in the $1^{\text {st }} 750$ group versus the effect size of if they had been randomly assigned to be in the $2^{\text {nd }} 750$ group. We dummy coded the data to which half group participants were in $\left[1^{\text {st }}\right.$ half $=1,2^{\text {nd }}$ half $\left.=0\right]$. To examine the effect of analyzing one half of the results before the other, labs were randomly assigned to analyze the $1^{\text {st }} 750$ first or second before aggregating the data into a complete $\mathrm{N} \gtrsim 1500$. Thus, we include a dummy variable in the metaregression for whether the $1^{\text {st }} 750$ was analyzed first or second $\left[1^{\text {st }}=1,2^{\text {nd }}=0\right]$. Although we did not predict an interaction between these two dummy variables, we included it anyway as an exploratory analysis. This model also included a random effect for each study and experiment nested within study.

As a specification check, we also estimated these effects using differences in effect sizes between sample halves. The main advantage of modeling the differences in effect sizes is that it requires weaker assumptions than fitting a model for the joint distribution of the effect size estimates. Thus, we subtracted the effect size from the $1^{\text {st }} 750$ group from the $2^{\text {nd }} 750$ group, so negative values of the variable would represent a decline in the magnitude of effect size from $1^{\text {st }}$ to $2^{\text {nd }}$ groups. In this model, the random effects terms now capture study-level and experimentlevel variation in the time-based decline effects and variation in the analysis order effects, rather than variation in the original effect size estimates.

\section{Slope Across Replications}


The third test looked at the change in effect size over time as replications accumulate for a given study. We further examined whether the change in effect size over time was moderated by whether the study (confirmation and replications) was blinded. If observer effects are the cause, we would expect the slope of the effect sizes to be moderated by whether a study was blind, with change being stronger in non-blind studies and blinded studies showing little or no change.

Thus, for a given study, coding the confirmation as 0 for the y-intercept and each replication in order as 1,2,3, and 4; fitting an individual-level growth curve to the change in effect size across replications for a given study would return a slope. Negative slopes would indicate a decline in effect sizes across replications, positive slopes would indicate an incline in effect sizes across replications, and a flat slope would indicate a stable effect.

We then tested the aggregate of these slopes for the 16 high-powered studies. As the order in which the replications were run was randomized via a Latin square design, if one lab consistently showed weaker effect sizes than the other labs, that effect would be spread out across replication orders, neutralizing any statistical leverage on the slope.

In this meta-regression model, we also included a dummy variable for whether the study was blinded. The prediction from this test was that blind studies would show a weaker or no decline in effect sizes while normal studies would show stronger decline ${ }^{8}$. The model also includes random effects for each confirmation or replication attempt of each study. The random effects were allowed to covary within study according to an auto-regressive structure.

\section{Pre-registered Analyses of Declining Effect Sizes}


We had three main analyses to test the replicability of new findings as well as testing declining effects. The first was that effect sizes would decline within laboratories between confirmatory study and self-replication. The second analysis concerns the 750/750 randomized splits. The third is the nature of effect sizes across laboratories and replications.

\section{General estimation methods}

All analyses were conducted using the R statistical computing environment (Version 4.2.1 $)^{9}$. All analyses used meta-analytic random effects models estimated using restricted maximum likelihood with the metafor package (Version 2.1.0) ${ }^{10}$. Standard errors and confidence intervals for all analyses were calculated using cluster-robust standard errors (CR2-type), clustering by study, using the clubSandwich package (Version 0.3.5) ${ }^{11}$. Hypothesis tests were based on Satterthwaite-type small-sample corrections to account for the limited number of independent studies.

\section{Studies}

The complete list of studies can be found in Table S2.

Table S2. Links to materials documenting all 16 confirmatory tests and replications, including data collection materials, data, analysis syntax, output, and report of findings, and discoveryoriented research, if any. Preregistration documents describe design and analysis plans. Order of Replication 1-4 matches the order the replications were run in (see Table S1).

\begin{tabular}{|c|c|c|c|c|c|}
\hline Study Name & $\begin{array}{l}\text { Confirmatory } \\
\text { Test }\end{array}$ & Replication 1 & Replication 2 & Replication 3 & Replication 4 \\
\hline Tumor & $\begin{array}{l}\text { Project: } \\
\underline{\text { https://osf.io/4n }} \\
\underline{8 \mathrm{pf} /}\end{array}$ & $\begin{array}{l}\text { Project: } \\
\text { https://osf.io/5 } \\
\text { ypsq/?view_o } \\
\text { nly=2870c6f1 } \\
\text { 8eda4a4c8d6c } \\
\underline{769366 \mathrm{ebf} 33 \mathrm{~d}}\end{array}$ & $\begin{array}{l}\text { Project: } \\
\text { https://osf.io/8d } \\
\underline{\text { e } 3 \mathrm{f} /}\end{array}$ & $\begin{array}{l}\text { Project: } \\
\text { https://osf.io/u8 } \\
\underline{\text { hq9/?view_only }} \\
\underline{=680 \mathrm{c} 20470 \mathrm{a} 90} \\
\underline{\text { 4cd8831182f7a }} \\
\underline{1595 \mathrm{f} 6 \mathrm{f}}\end{array}$ & $\begin{array}{l}\text { Project: } \\
\text { https://osf.io/u } \\
\underline{\text { tck4/ }}\end{array}$ \\
\hline
\end{tabular}




$\begin{array}{lll} & \begin{array}{l}\text { Prereg: } \\ \text { https://osf.io/z }\end{array} & \\ \text { Prereg: } & \underline{\text { 9kuz }} & \text { Prereg: } \\ \text { https://osf.io/zm } & & \underline{\text { https://osf.io/k6 }} \\ \underline{\text { 87e }} & \underline{\underline{2 n h}}\end{array}$

Prereg: https://osf.io/58 $\underline{\mathrm{n} 4 \mathrm{~b}}$

Project:

https://osf.io/jr9

$\mathrm{pc} /$

$$
\underline{\operatorname{adg} /}
$$

Prereg: https://osf.io/txj 9e

Project: Project:

Cookies

Label https://osf.io/y 7u5v/?view_o $\underline{\text { nly }=31 \text { be } 66 \mathrm{~b} 5}$ 25544598b7c $\underline{029 \mathrm{e} 273 \mathrm{bb} 45}$ $\underline{2 a}$

https://osf.io/8k c59/?view_only $=\mathrm{b} 8 \mathrm{a} 9 \mathrm{beddea} 0 \mathrm{c}$ 4d899739503eb 6c516f5

Prereg:

Prereg: $\quad$ https://osf.io/hw

https://osf.io/k $\underline{5 \mathrm{p} 4 \mathrm{f}}$
Project: Project:

https://osf.io/5

$\underline{\mathrm{h} 2 \mathrm{gw} /}$
Prereg: $\quad$ Prereg:

https://osf.io/sd https://osf.io/u zh5 ytk9
Prereg:

https://osf.io/y agxp

Project:

https://osf.io/k

zwa6/
Project: https://osf.io/8x dwcl https://osf.io/2n $\underline{\mathrm{tf} 3 /}$
Project: https://osf.io/dc 4xm/
Project:

https://osf.io/3 vz4k/?view_o $\underline{\text { nly }=\text { da10896b }}$ $\underline{68 \mathrm{fe} 4420 \mathrm{bf} 6 \mathrm{c}}$ $\underline{65 a 3 a 7 b d 64 f 6}$

Prereg:

\begin{tabular}{|c|c|c|c|c|}
\hline $\begin{array}{l}\text { Prereg: } \\
\text { https://osf.io/74 } \\
\end{array}$ & $\begin{array}{l}\text { Prereg: } \\
\text { https://osf.io/7 } \\
\end{array}$ & $\begin{array}{l}\text { Prereg: } \\
\text { https://osf.io/v9 }\end{array}$ & $\begin{array}{l}\text { Prereg: } \\
\text { https://osf.io/tn }\end{array}$ & $\begin{array}{l}\text { Prereg: } \\
\text { https://osf.io/v } \\
\text { mcy2 }\end{array}$ \\
\hline vu2 & $2 x g d$ & 658 & kw9 & \\
\hline
\end{tabular}

Project: Project: Project: Project: Project: https://osf.io/f5z https://osf.io/a https://osf.io/zm https://osf.io/w https://osf.io/j $\underline{\mathrm{dr} /}$

kn6/?view_only n9af/ $=\mathrm{c} 77 \mathrm{fbee} 4 \mathrm{f} 1 \mathrm{~b} 2$ 40a2837849912 $\underline{\text { dadbe60 }}$

Prereg:

Prereg:
https://osf.io/dw $\underline{3 \mathrm{fm}}$
Prereg: https://osf.io/z yst7 https://osf.io/7n

9yg
Prereg: https://osf.io/n4 $\underline{\mathrm{fr}}$ q64y/?view_o $\underline{\text { nly }=3421 \mathrm{e} 6 \mathrm{ac}}$ 51c642b98c5d cf9507cd27ea

Prereg: https://osf.io/r $\underline{\operatorname{td} 9 \mathrm{~b}}$ 


\begin{tabular}{|c|c|c|c|c|c|}
\hline \multirow[t]{8}{*}{ Self-control } & $\begin{array}{l}\text { Project: } \\
\text { https://osf.io/h2 }\end{array}$ & $\begin{array}{l}\text { Project: } \\
\text { https://osf.io/x }\end{array}$ & $\begin{array}{l}\text { Project: } \\
\text { https://osf.io/pk }\end{array}$ & $\begin{array}{l}\text { Project: } \\
\text { https://osf.io/fb }\end{array}$ & $\begin{array}{l}\text { Project: } \\
\text { https://osf.io/5 }\end{array}$ \\
\hline & $\overline{\mathrm{pwm} /}$ & jas9/?view_on & $\overline{\text { uv9/?view_only }}$ & nkg/ & xqya/?view_o \\
\hline & & $1 y=9 c 94526 a 2$ & $=83 \mathrm{~d} 22 \mathrm{ae} 30533$ & & $\mathrm{nly}=63 \mathrm{fedd} 7 \mathrm{e}$ \\
\hline & & de24ba8b8a7 & $439 \mathrm{ab} 7 \mathrm{dd} 33 \mathrm{~b} 7 \mathrm{c}$ & & $94964 d 5 a 814$ \\
\hline & & $\overline{96 \mathrm{e} 2 \mathrm{e} 9 \mathrm{e} 9 \mathrm{a00}} \mathrm{f}$ & 7595522 & & $\overline{d 94 c d 073 b 29}$ \\
\hline & & & Prereg. & & $\underline{35}$ \\
\hline & Prereg: & Prar & https://osf.io/v2 & Prereg: & Prereg: \\
\hline & 9ha & $\underline{\text { https://osf.io/q }}$ & q] & $\underline{\mathrm{ud}}$ & pucv \\
\hline \multirow[t]{7}{*}{ Orientation } & $\begin{array}{l}\text { Project: } \\
\text { https://osf.io/s6q }\end{array}$ & $\begin{array}{l}\text { Project: } \\
\text { https://osf.io/5 }\end{array}$ & $\begin{array}{l}\text { Project: } \\
\text { https://osf.io/pd }\end{array}$ & $\begin{array}{l}\text { Project: } \\
\text { https://osf.io/t6 }\end{array}$ & $\begin{array}{l}\text { Project: } \\
\text { https://osf.io/r }\end{array}$ \\
\hline & $\underline{\mathrm{dv} /}$ & ygj8/ & $\underline{4 \text { s9/?view only }}$ & $2 \mathrm{bd} /$ & tb34/?view_o \\
\hline & & & $=97405 \mathrm{c} 457 \mathrm{~b} 83$ & & $\underline{\text { nly }}=\mathrm{d} 00211 \mathrm{f} 1$ \\
\hline & & & 49ef844196ee2 & & $229 \mathrm{c} 4635 \mathrm{~b} 69 \mathrm{a}$ \\
\hline & & & $\underline{67 \mathrm{e} 795 \mathrm{~b}}$ & & $84444 \mathrm{~cd} 72 \mathrm{f} 08$ \\
\hline & Prereg: & Prereg: & $\begin{array}{l}\text { Prereg: } \\
\text { https://osf.io/8n }\end{array}$ & Prereg: & $\begin{array}{l}\text { Prereg: } \\
\text { https://osf.io/f }\end{array}$ \\
\hline & $\underline{\text { https://ost.10/54t }}$ & $\underline{\text { https://ost.10/x }}$ & $\underline{\mathrm{zWm}}$ & $\frac{\text { https://Osf.10/X3 }}{\text { pjn }}$ & 9yng \\
\hline \multirow[t]{7}{*}{ Referrals } & $\begin{array}{l}\text { Project: } \\
\text { https://osf.io/v3t }\end{array}$ & $\begin{array}{l}\text { Project: } \\
\text { https://osf.io/b }\end{array}$ & $\begin{array}{l}\text { Project: } \\
\text { https://osf.io/wt }\end{array}$ & $\begin{array}{l}\text { Project: } \\
\text { https://osf.io/e5 }\end{array}$ & $\begin{array}{l}\text { Project: } \\
\text { https://osf.io/j }\end{array}$ \\
\hline & $\underline{\mathrm{hd} /}$ & $\begin{array}{l}\text { sg7f/?view_o } \\
\text { nly=9129739 }\end{array}$ & $\underline{\mathrm{z} 9 \mathrm{~g} /}$ & $\begin{array}{l}\text { u42/?view_only } \\
=1 \mathrm{c} 32808 \mathrm{a} 01 \mathrm{ee}\end{array}$ & epfa/ \\
\hline & & $\underline{897 b 04 a 4 b a 6}$ & & $4 \mathrm{c} 8 \mathrm{c} 816480825$ & \\
\hline & & 73297a9e3e6c & & ad5bebf & \\
\hline & & $\underline{\mathrm{e} 4}$ & & & \\
\hline & Prereg: & Prereg: & Prereg: & $\underline{\text { https://osf.io/pjr }}$ & Prereg: \\
\hline & w62 & $\frac{\text { https://osf.10/q }}{6 \mathrm{rnz}}$ & $\begin{array}{l}\text { ntups://OSI.10/qX } \\
\text { gwm }\end{array}$ & $\underline{9 c}$ & $\underline{\text { he } 3 \mathrm{~h}}$ \\
\hline \multirow[t]{5}{*}{ Ads } & $\begin{array}{l}\text { Project: } \\
\text { https://osf.io/yh }\end{array}$ & $\begin{array}{l}\text { Project: } \\
\text { https://osf.io/z }\end{array}$ & $\begin{array}{l}\text { Project: } \\
\text { https://osf.io/zq }\end{array}$ & $\begin{array}{l}\text { Project: } \\
\text { https://osf.io/tx }\end{array}$ & $\begin{array}{l}\text { Project: } \\
\text { https://osf.io/r }\end{array}$ \\
\hline & $\underline{\mathrm{u}} 44 /$ & c8p9/ & $\underline{8 g y /}$ & $\underline{\text { me4/?view_onl }}$ & pxa8/?view_o \\
\hline & & & & $\mathrm{y}=8 \mathrm{cf} 18 \mathrm{a} 2 \mathrm{babc}$ & $\underline{\text { nly }=4 \mathrm{a} 111 \mathrm{fdb}}$ \\
\hline & & & & $\underline{1499 \mathrm{e} 98 \mathrm{ef} 57 \mathrm{db}}$ & $\underline{\mathrm{e} 8 \mathrm{ef} 404 \mathrm{aa} 80 \mathrm{~d}}$ \\
\hline & & & & b9926a80 & cf83c9ac1fa7 \\
\hline
\end{tabular}


Prereg: Prereg: Prereg: Prereg:

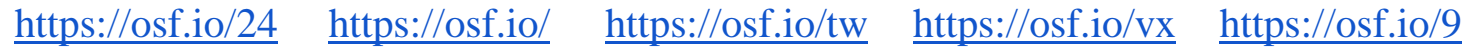
$\underline{\text { ndc }} \quad \underline{\operatorname{myx} 9 \mathrm{c}} \quad \underline{9 \mathrm{rh}} \quad \underline{\mathrm{kep}} \quad \underline{\mathrm{u} 2 \mathrm{nx}}$

Fast Social Project: Project: Project: Project: Project:

Desirability https://osf.io/yx https://osf.io/j https://osf.io/m https://osf.io/du https://osf.io/2 (FSD)

$\underline{\text { wc3/ }} \begin{array}{ll}\text { se68/ } & \frac{\text { 6avf/?view_onl }}{\text { y=fda7fcac8d75 }} \\ \frac{\text { 4d4bbe033ec34 }}{\text { a134414 }}\end{array}$

gteq/?view_on $\underline{\mathrm{y}}=8 \mathrm{f} 38 \mathrm{e} 1 \mathrm{ada}$ $\underline{\text { ba149cb8bb1f }}$ $\underline{\text { b1477a759d9 }}$

\begin{tabular}{|c|c|c|c|c|}
\hline $\begin{array}{l}\text { Prereg: } \\
\text { https://osf.io/zac } \\
\text { b3 }\end{array}$ & $\begin{array}{l}\text { Prereg: } \\
\text { https://osf.io/6 } \\
3 \mathrm{z} 4 \mathrm{~g}\end{array}$ & $\begin{array}{l}\text { Prereg: } \\
\text { https://osf.io/yu } \\
\underline{8 \mathrm{hw}}\end{array}$ & $\begin{array}{l}\text { Prereg: } \\
\text { https://osf.io/b2 } \\
489\end{array}$ & $\begin{array}{l}\text { Prereg: } \\
\text { https://osf.io/v } \\
\underline{\underline{3 e x z}}\end{array}$ \\
\hline
\end{tabular}

Prediction Project: Project: Project: Project:

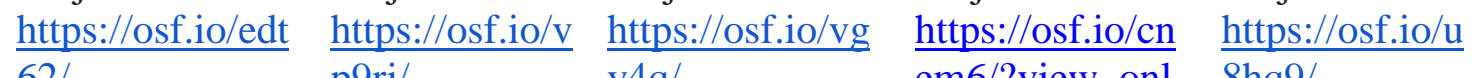
62/ p9rj/ y4q/ em6/?view_onl $\underline{8 \text { hq9/ }}$ $\mathrm{y}=\mathrm{e} 458 \mathrm{fc} 4 \mathrm{deb} 3$ 94549ab532dc9 ef647f1e

\begin{tabular}{|c|c|c|c|c|}
\hline $\begin{array}{l}\text { Prereg: } \\
\text { https://osf.io/yrx } \\
2 t\end{array}$ & $\begin{array}{l}\text { Prereg: } \\
\text { https://osf.io/y } \\
\mathrm{s} 62 \mathrm{a}\end{array}$ & $\begin{array}{l}\text { Prereg: } \\
\text { https://osf.io/3g } \\
\text { vah }\end{array}$ & $\begin{array}{l}\text { Prereg: } \\
\text { https://osf.io/nc } \\
\underline{2 \mathrm{t} 9}\end{array}$ & $\begin{array}{l}\text { Prereg: } \\
\text { https://osf.io/5 } \\
8 \mathrm{n} 4 \mathrm{~b}\end{array}$ \\
\hline
\end{tabular}

Fairness Project: Project: Project: Project:

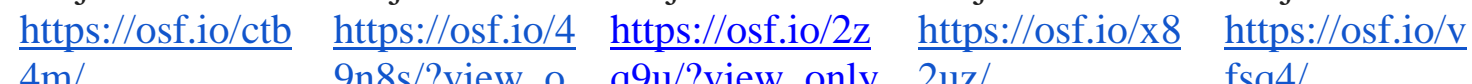
$\underline{4 \mathrm{~m} /}$ 9n8s/?view_o q9u/?view_only $\underline{\mathrm{nly}=81819 \mathrm{e} 97 \quad \equiv \mathrm{c} 8 \mathrm{c} 0 \mathrm{e} 29 \mathrm{dbd} 22}$ $\underline{\mathrm{uzl}} \quad \underline{\mathrm{fsq} 4 /}$ $\underline{\mathrm{d} 13 \mathrm{e} 4099 \mathrm{~b} 3 \mathrm{c}} \quad \underline{48 \mathrm{db} 8 \mathrm{dbb} 25543}$ 2c94052efefd $\underline{58 \text { be61c }}$ $\underline{4}$

Prereg: $\quad$ Prereg: $\underline{\text { https://osf.io/63 }}$ $\underline{\mathrm{x}} \mathrm{k} \mathrm{k}$ $\underline{\underline{\text { myj7 } 7}}$ Prereg: Prereg: https://osf.io/52 https://osf.io/z $\underline{\text { z34 }} \quad \underline{\text { cusf }}$ 


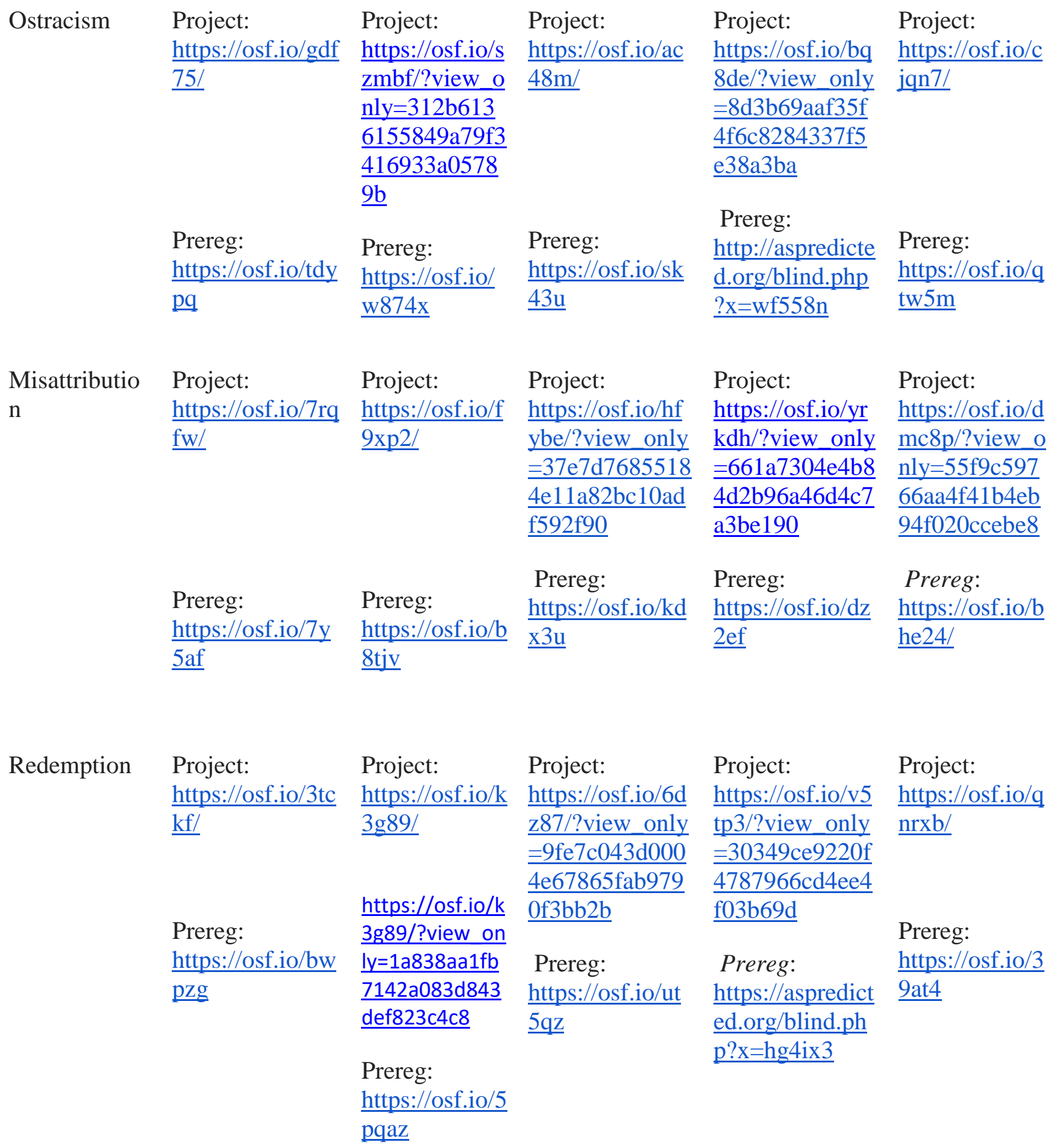




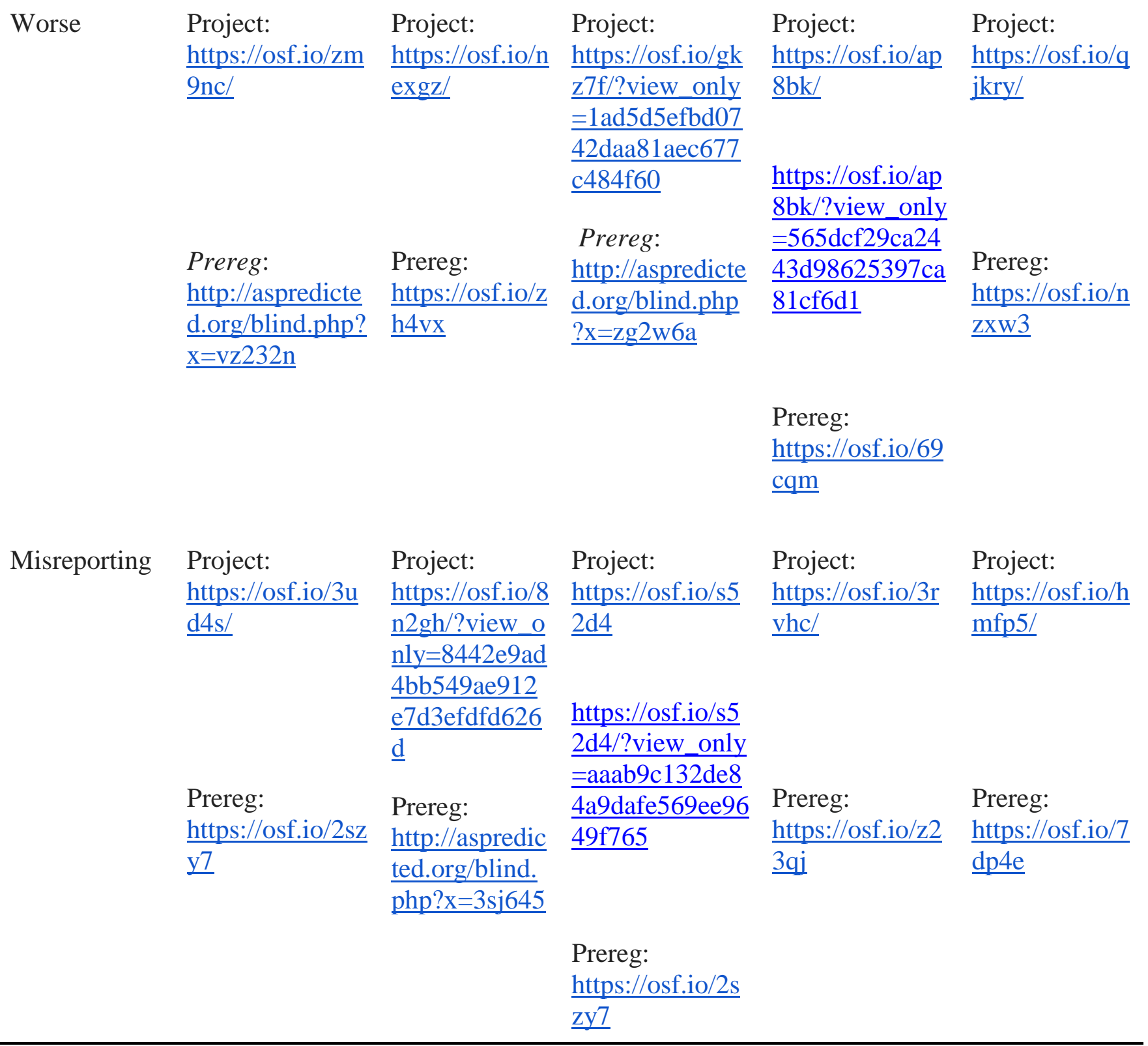

\section{Results}

\section{SI Study 1: Predictability of Study Outcomes by Practicing Scientists}

To gauge how predictable the results of the confirmation studies were for practicing scientists in a range of fields, we conducted a survey of attendees to the Metascience 2019 
conference held September 5-8, 2019 at Stanford University. Email invitations were sent out on September 4, 2019 to all who registered, said they would attend the conference, and provided their email address $(N=494)$. Attendees had a wide range of research experience, from undergraduates to tenured professors, in fields such as Psychology, Medicine, Philosophy, Sociology, Communication, Political Science, Biology, Physics, Statistics, Economics. 68\% of the people who answered had a Ph.D. (27\% in Psychology, $73 \%$ in a different field). Of those who did not have a Ph.D., $41 \%$ were enrolled in a graduate program pursuing a Ph.D. in psychology. The survey was approved by the Stanford University Institutional Review Board.

Participants were told: "Here are the descriptions of 15 experiments that were recently run. Each study involved two between-subjects conditions with 750 participants in each condition. After you read each description, you will predict the results that might be observed." Participants then read a brief description of 12 of the 16 experiments (descriptions of four of the studies were not obtained before the survey invitations were sent). Each account described two conditions (condition A and condition B) to which participants had been randomly assigned. One or two sentences described what participants were asked to do in both experimental conditions. The description of each study was submitted by the lab creating the study and modified by experts in question wording and survey methodology for clarity and completeness. An example, chosen because prediction rates for this study most closely matched the average prediction success across all participants, is:

Condition A: Participants read about an individual being attacked by someone who had low self-control because of a brain injury.

Condition B: Participants read about an individual being attacked by someone who had low self-control because of his/her genes.

DV: Participants decided whether the attacker should be found guilty of assault and battery. 
After reading such a description of each study, participants were asked: "Please indicate the percent chance that each of these three possible outcomes will be the result of this study." They were given three options: that the dependent variable was higher in Condition A than Condition B, that the dependent variable was higher in Condition B than in Condition A, and that the dependent variable was not significantly different in the two conditions. These response options were customized to each study. For the example problem described above, the response options were:

- Significantly more participants in Condition A indicated "guilty" than in Condition B

- Significantly more participants in Condition B indicated "guilty" than in Condition A

- The number of participants in the two conditions who indicated "guilty" was not significantly different from one another

Participants were asked: "Please indicate the percent chance that each of these three possible outcomes will be the result of this study. Please type numbers between zero percent and 100\%, and your three answers must add up to $100 \% . "$

In addition to the 12 confirmatory test descriptions, three other descriptions were provided, from pilot studies that had been run but yielded non-significant treatment effects. These studies also involved two between-subjects conditions. Data collection ended two days after the link was sent out and the results of the survey were incorporated into a presentation at the conference. This survey was preregistered prior to data collection. Information about the survey is at https://osf.io/3yhbe/, where all study descriptions can be found. Data collection was stopped on September 8, 2019 before a presentation of the study on which this survey was based. 72 people completed the questionnaire, yielding a response rate of $14.6 \%$. 
Results of the survey indicate the 'surprisingness' of the results of the studies in this meta-analysis. Expert scientists were able to predict the correct response only $42 \%$ of the time. Interestingly, participants incorrectly predicted individual studies producing a statistically significant effect would not $38 \%$ of the time. And, $20 \%$ of the time, participants predicted significant treatment effects in the opposite direction of what was actually observed.

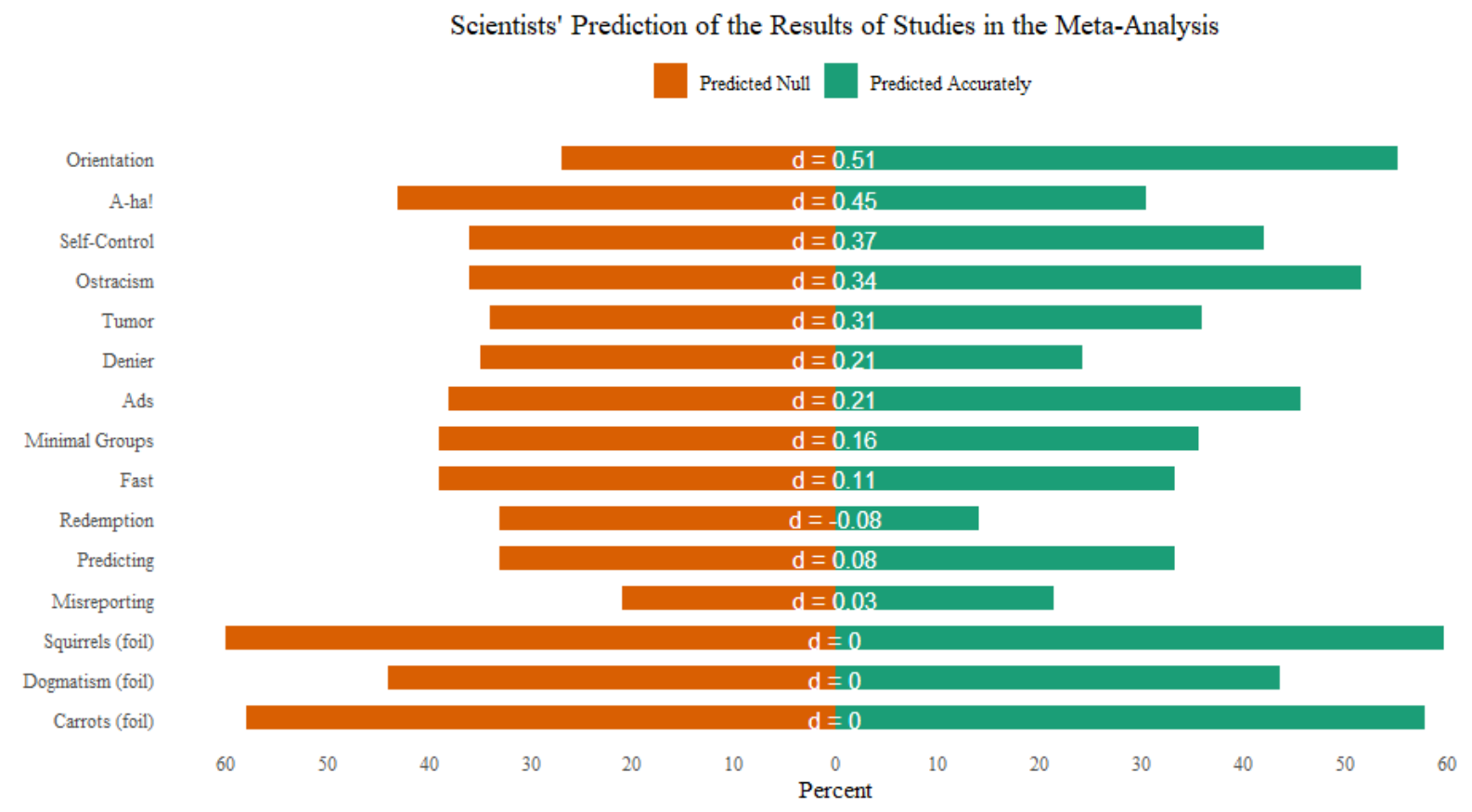

Fig S1: Percent of scientists surveyed and asked to predict the results of 12 of the studies in this meta-analysis plus three foils. Bars on the right show participants who predicted the outcome accurately. Bars on the left show participants who predicted there would be no significant difference between the groups. Labels inside the bars are the absolute value of treatment effect sizes from the Confirmatory tests. The Spearman correlation between prediction accuracy and observed effect size was .104, $p=.712$ ).

\section{SI Study 2: Predictability of Study Outcomes Compared with Similar Findings that}

\section{Showed Low Replicability}

To gauge whether the results of the confirmation studies were unusually obvious, we recruited respondents via Prolific and asked them to predict the findings of our confirmation 
studies that showed high replicability and the findings from a previous large-scale multi-lab replication project in the social-behavioral sciences (referred to as the "comparison effects") ${ }^{1,3}$ that showed much lower replicability. If our highly replicable findings are likewise much easier to predict than less replicable findings, then our findings may be unusually "trivial" in comparison to other social-behavioral research. That could mean that our high replicability is more a consequence of choosing obviously true findings rather than introduction of rigorenhancing practices.

Participants answered six prompts, three of which were sampled at random from among the 16 effects identified in the confirmation studies and three of which were sampled at random from among 20 comparison effects. For each prompt, we recorded:

1. the respondent's prediction regarding the sign of the effect;

2. the respondent's rating of their level of understanding from 1 ("Not at all well") to 5 ("Very well");

3. the respondent's confidence rating for their prediction, from 1 ("Not at all sure") to 5 ("Extremely sure");

4. response time (in seconds) to the sign prediction item; and

5. The respondent's prediction regarding whether the effect would replicate (yes or no).

Our primary aim was to test the equivalence between the rate of correct sign predictions for effects in the present project and the rate of correct sign predictions for the comparison effects. Specifically, we designed the study to the focal null hypothesis that the average predictability of the confirmation study effects exceeds the average predictability of the comparison effects by a threshold of 5 percentage points or greater, using the conventional alpha level of .05 . We tested our focal hypothesis by directly estimating predictability rates of each effect, using the results to calculate the average predictability of both sets of effects, and then testing whether the difference between the confirmation study effect and comparison effects is 
no more than 5 percentage points. For consistency with the (one-sided) equivalence test, we reported $90 \%$ confidence intervals.

To determine an adequate sample size for the test, we used Monte Carlo simulation to generate data under a normal ogive item response model and conservative assumptions about the variation in participants' prediction abilities and variation in the predictability of each set of prompts. Based on these assumptions and a goal of $95 \%$ power if the two sets of studies had identical predictability, we set a target sample size of $\mathrm{N}=1200$ respondents (after excluding any ineligible respondents.

We planned to exclude respondents if they met any of the following criteria: a) reported holding a Ph.D. in Psychology $(N=14)$, b) failed the Captcha item at the end of the survey $(N=$ $0), \mathrm{c})$ indicated at the end of the survey that they did not provide serious responses $(N=13$ agreeing with "I have just clicked through, please throw my data away."), or d) had an average level of understanding of 2.0 ("slightly") or less $(N=16)$. Additionally, we excluded responses to specific prompts if the respondent indicated the lowest level of understanding ("Not at all well”). This resulted in exclusion of an additional 67 responses from 62 unique respondents.

The survey design and analysis plan was pre-registered prior to data collection beginning. The preregistered analytic plan, survey materials, data, and complete results are available at https://osf.io/43fn7/. Data collection on Prolific occurred on December 12, 2022.

We obtained a total of 1223 partial or complete responses to the survey. After applying all exclusion criteria, the analytic sample included $N=1180$ participants. About $55 \%$ of respondents held a bachelor's degree or higher degree; $1 \%$ were currently enrolled in a doctoral 
program in Psychology. About 56\% reported having taken a college or university course in Psychology.

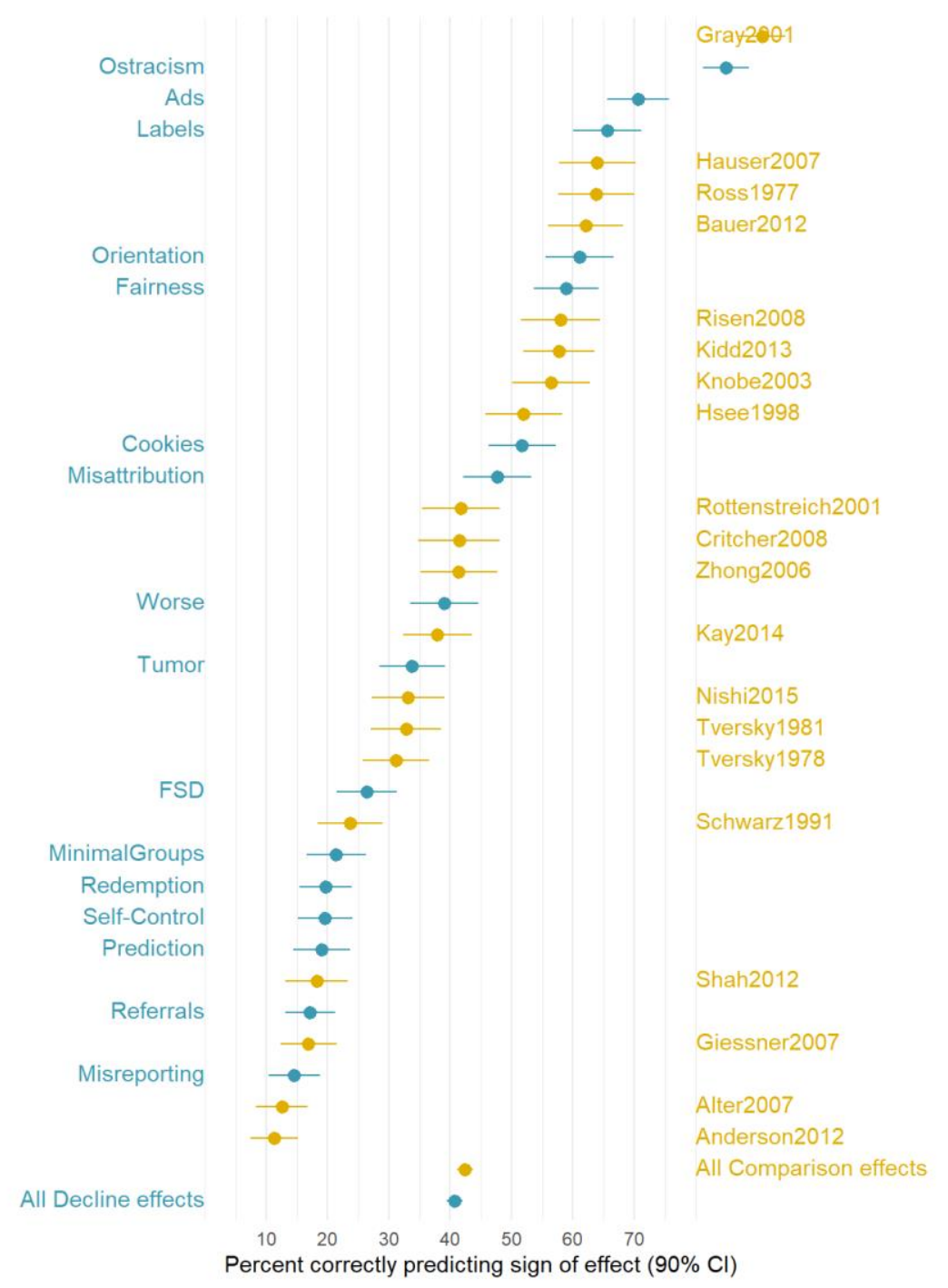

Our primary outcome analysis examined whether respondents could correctly predict the sign of the effect from a written description. The figure above depicts the percentage of correct predictions by effect, ordered from most to least predictable. We estimated that the average predictability of confirmation study effects differed from the predictability of the comparison effects by -1.6 percentage points, $90 \%$ CI: $[-3.4,0.1]$. Under the null of a 5 percentage point difference, this result corresponds to $\mathrm{p}<.0001$. 
As a secondary outcome, we repeated the analysis using directional confidence scores, calculated by multiplying the self-reported confidence level (shifted by 1 ) by -1 if the respondent's prediction for sign of the effect was incorrect and +1 if the respondent's prediction was correct. Following the same analytic approach as for the primary outcome, we estimate that the average directional confidence score of confirmation study effects differed from that of the comparison effects by -0.04 points, $90 \%$ CI: [-0.12, 0.04]. Participants reported having a very similar level of understanding for the confirmation study effects and the comparison effects. Average response times to the confirmation study prompts were approximately 1 second longer than response times to comparison prompts. In pre-planned sensitivity analyses, we found that all results were robust to the exclusion of 410 item responses from $N=281$ participants who rated understanding the item "slightly" and to the exclusion of $N=14$ respondents with a median response time of 4 seconds or less.

One potential concern with this analysis is that the two sets of effects might have similar levels of predictability because participants were inattentive or put little cognitive effort into making predictions. If inattentiveness or low-effort responding were the explanation, we would expect to see little variation across effects in the percentage of respondents who correctly predict the true sign (i.e., all effects would be predicted at near-chance levels). We used an item response theory (IRT) model to investigate this potential alternative explanation by fitting a 1-parameter, normal ogive IRT model to the binary indicator for whether each participant correctly predicted the sign of each effect. This model allows participants to vary in their predictive skill and effects to vary in their predictability. Using REML estimation, we estimated between-participant variance of 0.02 and between-effect variance of 0.37 . The between-effect variance was significantly different from null $\left(\chi^{2}(1)=1200, p<.0001\right)$. 
If the effects vary in their degree of predictability, then one might expect that prompts describing larger effect sizes would be more predictable. Using the pooled effect size estimates from multi-lab replications of each finding, we examined whether the absolute magnitude of effect sizes was associated with predictability within the confirmation study effects and the comparison effects. Based on the 1-parameter normal ogive IRT model, clear associations were evident for the confirmation study prompts $(\beta=2.80, p=.003)$ and comparison study prompts ( $\beta=0.66, p=.002$ ). Absolute effect size magnitude explained approximately $35 \%$ of the between-effect variance in predictability.

This evidence is consistent with our interpretation that the newly discovered findings in this project that demonstrated high replicability are no more predictable or obvious in advance than prior discoveries that demonstrated low replicability. We believe that the reason for higher replicability is the adoption of rigor-enhancing practices to improve the likelihood of discovering replicable findings, and conducting high-quality replications of those findings. A detailed report of all analyses for this study is available included as an Appendix to the SI and is at https://osf.io/43fn $7 /$.

\section{Additional Results for the Study Described in the Main Text}

\section{Power}

For all 16 confirmatory tests, the average power was 0.802 , with a median of 0.993 and a range of 0.000 to 1.000 . The observed replication rate of $90 \%$ in the 64 replication attempts was slightly larger than the expected positive result rate based on power estimates. This could occur if some of the confirmatory tests underestimated their true effect sizes, as appeared to be the case for two of the three confirmatory tests that showed null results but had statistically significant 
treatment effects in the expected direction for most or all of the replication studies. Three of the confirmatory tests produced evidence consistent with the null hypothesis. These were the 'Prediction' study $(\mathrm{BF}=5.166)$, 'Redemption' $(\mathrm{BF}=5.981)$, and 'Misreporting' $(\mathrm{BF}=14.673$; Bayes Factors produced by using a naive prior and .707 scaling factor and are meant only for exploratory purposes ${ }^{12}$ ). Aggregating the self-confirmatory test with their own replications for these studies suggests a small but reliable treatment effect in the expected direction for 2 of those 3 null confirmatory tests.

\section{Alternate Metric of Replicability}

A common metric to assess replicability is whether the $95 \% \mathrm{CI}$ between an original study and its replication overlap. This was used in the 100-study Reproducibility Project: Psychology ${ }^{1}$, for example. Estimating the 95\%CI of the self-confirmatory test ESs, 49/64 (77\%) replications ESs fell within those intervals, 15/16 (94\%) self-replications, 34/48 (71\%) independent replications. Thus, using 95\%CI overlap, treating the replication ESs as point estimates with no sampling error, produced smaller replication success rates.

\section{Testing whether effects decline}

\section{Confirmation Versus Self-Replication}

A first test of unusual possible explanations for declining effect sizes is to compare the effect size of a labs' confirmation studies versus the corresponding self-replications. To test this within-effect decline, we analyzed differences between the self-confirmation study and the selfreplication of the same effect by the same lab. A negative average change would be evidence of within-lab decline effects. We predicted that the more studies run between the confirmation 
study and the self-replication, the greater the decline. Finally, we would expect to see larger declines in non-blinded studies than in blinded studies.

First, we calculated differences in standardized effect sizes, pooling effect size estimates across the two half-samples from each replication, taking

$$
d_{S j k}=\frac{1}{2}\left(d_{1 R_{j k} j k}+d_{2 R_{j k} j k}\right)-\frac{1}{2}\left(d_{10 j k}+d_{20 j k}\right)
$$

with standard error given by

$$
\sigma_{S j k}=\frac{1}{2} \sqrt{\sigma_{1 R_{j k} j k}^{2}+\sigma_{2 R_{j k} j k}^{2}+\sigma_{10 j k}^{2}+\sigma_{20 j k}^{2}}
$$

Let $\tilde{R}_{j k}=R_{j k}-\frac{1}{16} \sum_{j=1}^{4} \quad \sum_{k=1}^{4} \quad R_{j k}$ be the grand-mean centered number of the selfreplication. We estimated the parameters of the following meta-regression equation:

$$
d_{S j k}=\beta_{0}+\beta_{1} \tilde{R}_{j k}+\beta_{2} B_{j k}+u_{j k}+e_{j k}
$$

where the sampling error term $\mathrm{e}_{\mathrm{jk}}$ is assumed to have known variance $\sigma_{S j k}^{2}$. Note that the random effect $\mathrm{u}_{\mathrm{jk}}$ captures between-study variation in within-lab decline effects. In this model, $\beta_{0}$ represents the average within-study, within-lab difference between self-replication experiment and confirmation experiment; $\beta_{1}$ represents the average exposure effect, which is the difference in within-study decline effects for self-replication studies conducted after one further intervening replication; and $\beta_{2}$ represents the difference in within-study decline effects between blinded and non-blinded studies. The hypothesis test for $\beta_{0}=0$ used $\alpha=.05$. The tests of $\beta_{1}$ and $\beta_{2}$ were treated as exploratory.

This analysis tested whether effect sizes declined in between a lab's self-confirmation and their own self-replication. This analysis holds all aspects of the lab constant. The only 
difference is the self-replication was run at a later time in a different group of participants drawn from the same population, with between 0 and 3 replications run in between the two.

In no case was there a statistically significant difference between the magnitude of the confirmation study effect size and the magnitude of the self-replication effect size. On average, self-replication effect sizes were the same size as the confirmation study sizes $(\mathbf{d}=-0.003, p=$ $.864,95 \% \mathrm{CI}=-0.035$ to 0.030$)$.

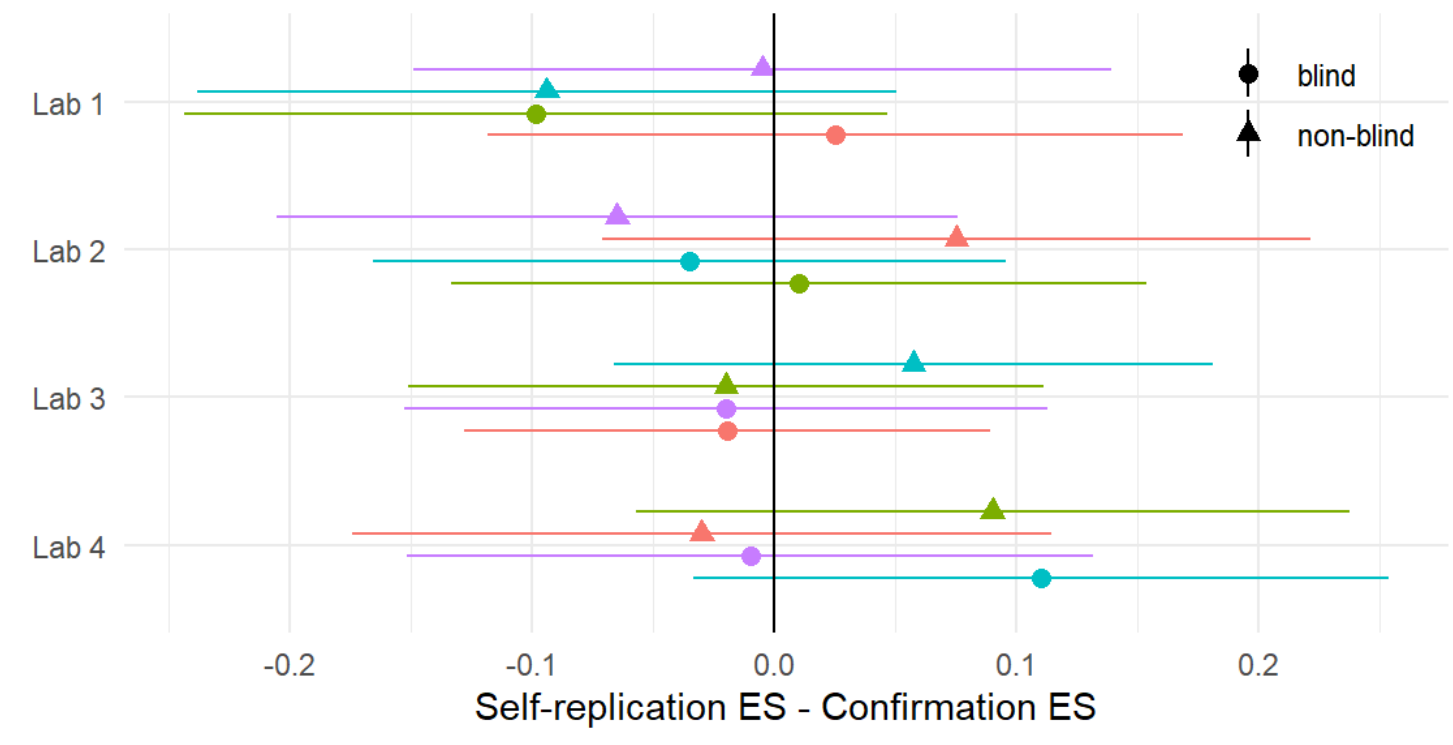

Fig S4: Difference between a confirmation study and the self-replication in SD units. Error bars represent $95 \%$ CIs.

There was also no effect of whether the studies were 'blind' $(b=-0.009, p=.760,95 \% \mathrm{CI}$ $=-0.074$ to 0.055$)$ nor the number of replications run in between the two $(b=-0.008, p=.435$, $95 \% \mathrm{CI}=-0.030$ to .015$)$. As the primary $\mathrm{DV}$ is not the replication effect size but the difference between self-confirmation and replications, all predictors are main effects, although the coefficients can be interpreted as though they were interactions had a full model (with replication ES as the DV and confirmation ES as a predictor) been run. Thus, when a lab replicated its own 
pre-registered study using the same pre-registered procedures, the two tests produced nearly identical effect sizes.

\section{Slope Across Replications}

The third test of unusual possible reasons for declining effects looks at the change in effect size over time as replications accumulate, and the interaction of such a decline with blinding. If observer effects cause declines in effect sizes, then we would expect the slope of the temporal decline to be greater in unblinded studies than in blinded studies, which might show little or no decline.

To examine temporal decline, we first aggregated effect size estimates across the halfsamples from each confirmation study and each of the replication studies. Let $d_{\bullet i j k}=$

$\frac{1}{2}\left(d_{1 i j k}+d_{2 i j k}\right)$, with standard error $\sigma_{\bullet i j k}=\frac{1}{2} \sqrt{\sigma_{1 i j k}^{2}+\sigma_{2 i j k}^{2}}$. To formally test for time trends across waves, we estimated the following meta-regression model based on the aggregated effect size estimates:

$$
d_{\bullet i j k}=\alpha_{k}+\beta_{1}(i)+\beta_{2} B_{j k}+\beta_{3}(i) B_{j k}+u_{i j k}+e_{i j k}
$$

where $\alpha_{k}$ is a fixed effect for each lab, representing the average effect size in confirmation studies originating from that lab, $\beta_{1}$ is the average change in effect size for each successive replication study, $\beta_{2}$ is the average difference in effect sizes between blinded studies and unblinded studies, and $\beta_{3}$ represents the difference in slopes between blinded and unblinded studies (i.e., the interaction between the temporal decline and blinding). The model also includes random effects for each confirmation or replication attempt of each study $\left(\mathrm{u}_{\mathrm{ijk}}\right.$ for $\mathrm{i}=0, \ldots, 4$; $\mathrm{j}=1, \ldots, 4 ; \mathrm{k}=1, \ldots, 4)$. The random effects are allowed to covary within study according to an auto- 
regressive structure, such that $\operatorname{Var}\left(\mathrm{u}_{\mathrm{ijk}}\right)=\tau^{2}, \operatorname{Cov}\left(\mathrm{u}_{\mathrm{ijk}}, \mathrm{u}_{\mathrm{i}^{\prime} \mathrm{jk}}\right)=\tau^{2} \rho^{\left|\mathrm{i}^{\prime}-\mathrm{i}\right|}$, and $\operatorname{Cov}\left(\mathrm{u}_{\mathrm{ijk}}, \mathrm{u}_{\mathrm{i}^{\prime} \mathrm{j}^{\prime} \mathrm{k}^{\prime}}\right)=0$ when $\mathrm{j} \neq \mathrm{j}^{\prime}$ or $\mathrm{k} \neq \mathrm{k}^{\prime}$. The sampling error term $\mathrm{e}_{\mathrm{ijk}}$ is assumed to have known variance $\sigma_{\bullet i j k}^{2}$.

We tested the hypothesis $\beta_{1}=0$ to examine temporal decline and $\beta_{3}=0$ to examine whether temporal declines are moderated by blinding. Hypothesis tests for $\beta_{1}$ and $\beta_{3}$ used testwise alpha levels of $\alpha=.025$ to control the family-wise error rate.

Across replications, there was no consistent change in effect size $(b=-0.002, p=.701$, $95 \% \mathrm{CI}=-0.015$ to 0.01$)$. These results do not change when removing the fixed effect for the lab and were the same for 'blind' and 'not blind' studies $(b=0.02, p=0.104)$.

Thus, preregistered and independent replications of preregistered novel findings produce treatment estimates that are stable over replications. Studies that are 'observed' before being replicated do not exhibit a statistically-significantly different slope of change at $p=.05$ (twotailed) than those that are kept blind.

\section{Lab-specific variation.}

In addition to testing decline, we also examined whether there is lab-specific variation in effect sizes, including variation across originating labs as well as which lab conducted a given replication. Questions about these sources of variation are ancillary to the tests of decline effects, and so we examined them in a separate family of hypothesis tests. We estimated the parameters of the following meta-regression equation:

$$
d_{\bullet i j k}=\alpha_{k}+\gamma_{L_{i j k}}+\beta_{1}(i)+\beta_{2} B_{j k}+\beta_{3}(i) B_{j k}+u_{0 j k}+(i) u_{1 j k}+e_{i j k}
$$

This model elaborates upon the previous model by including fixed effects $\gamma_{L_{i j k}}$ for the lab conducting the replication experiment. In the event of non-convergence, we planned to re- 
estimate the model after constraining random effects variance components to zero as necessary to achieve convergence, but this was not necessary.

We then tested two hypotheses pertaining to the originating lab effects and the replication lab effects. First, we tested the hypothesis $\alpha_{1}=\alpha_{2}=\alpha_{3}=\alpha_{4}$ to examine whether average effect sizes of the confirmation studies differed across labs. Second, we examined whether average effect sizes varied depending on the lab conducting the replication study by testing the hypothesis $\gamma_{1}=\gamma_{2}=\gamma_{3}=\gamma_{4}$. We tested these hypotheses using likelihood ratio tests (i.e., using model-based methods, rather than robust variance estimation) because of their greater power. We also conducted corresponding tests based on robust variance estimation methods (i.e., robust Approximate Hotelling's $\mathrm{T}^{2}$ tests) as sensitivity analyses.

\begin{tabular}{lllllll} 
& \multicolumn{2}{c}{ Likelihood Ratio } & & \multicolumn{3}{c}{ Approx. Hotelling's T-squared } \\
\cline { 2 - 3 } Effects & Chi-square & p-value & & F statistic & Denominator d.f. & p-value \\
\hline Originating lab effects & 2.94 & 0.40061696 & 0.65 & 5.85 & 0.611535 \\
\hline Replication lab effects & 20.18 & 0.00015557 & 5.18 & 12.26 & 0.015406
\end{tabular}

As a further sensitivity analysis, we re-estimated the model after removing the occasion predictor, the blinding indicator, and their interaction, as well as simplifying the random effects structure to a study-specific intercept, leaving:

$$
d_{\bullet i j k}=\alpha_{k}+\gamma_{L_{i j k}}+u_{0 j k}+e_{i j k}
$$

We then repeated the above hypothesis tests under the reduced model.

\begin{tabular}{|c|c|c|c|c|c|}
\hline \multirow[b]{2}{*}{ Effects } & \multicolumn{2}{|c|}{ Likelihood Ratio } & \multicolumn{3}{|c|}{ Approx. Hotelling's T-squared } \\
\hline & Chi-square & p-value & F statistic & Denominator d.f. & p-value \\
\hline Originating lab effects & 2.61 & 0.45556 & 0.70 & 6.01 & 0.586202 \\
\hline Replication lab effects & 34.82 & $<0.0001$ & 3.87 & 12.31 & 0.036972 \\
\hline
\end{tabular}


750/750 Split Sample Halves

The final test of usual possible reasons for declining effects assessed time-based decline within each experiment, observer effects, and their interaction. We made three predictions:

Time-based decline. Randomly assigning participants to two different half samples allows for a test of the hypothesis that effect sizes of experiments decline over time, with the main difference between the two samples being time of collection. That is, as participants were randomly assigned to the first or second period of data collection, we can test for a causal, timebased decline. We predicted effect sizes to be smaller in the second 750 participants than in the first 750 participants.

Observer effects: analysis order. To test for observer effects, labs were assigned to analyze the first 750 sample or the second 750 sample in a random order. The hypothesis that observation would impact effect sizes leads to the prediction that the 750 that was analyzed first would have a larger effect size than the 750 analyzed second. Including this analysis provides the opportunity to fail to observe evidence for exotic interpretations of declining effect sizes.

Interaction. We included an interaction term in the model of both observer effect order and data collection order.

Fig S6 below depicts the effect size estimates from the first and second half-samples of each experiment (including the initial confirmation study and subsequent replications), with separate plots for each of the originating labs. Each study is represented in a different color. If decline occurred, the effect size estimates would tend to fall in the lower triangle of the plot. 


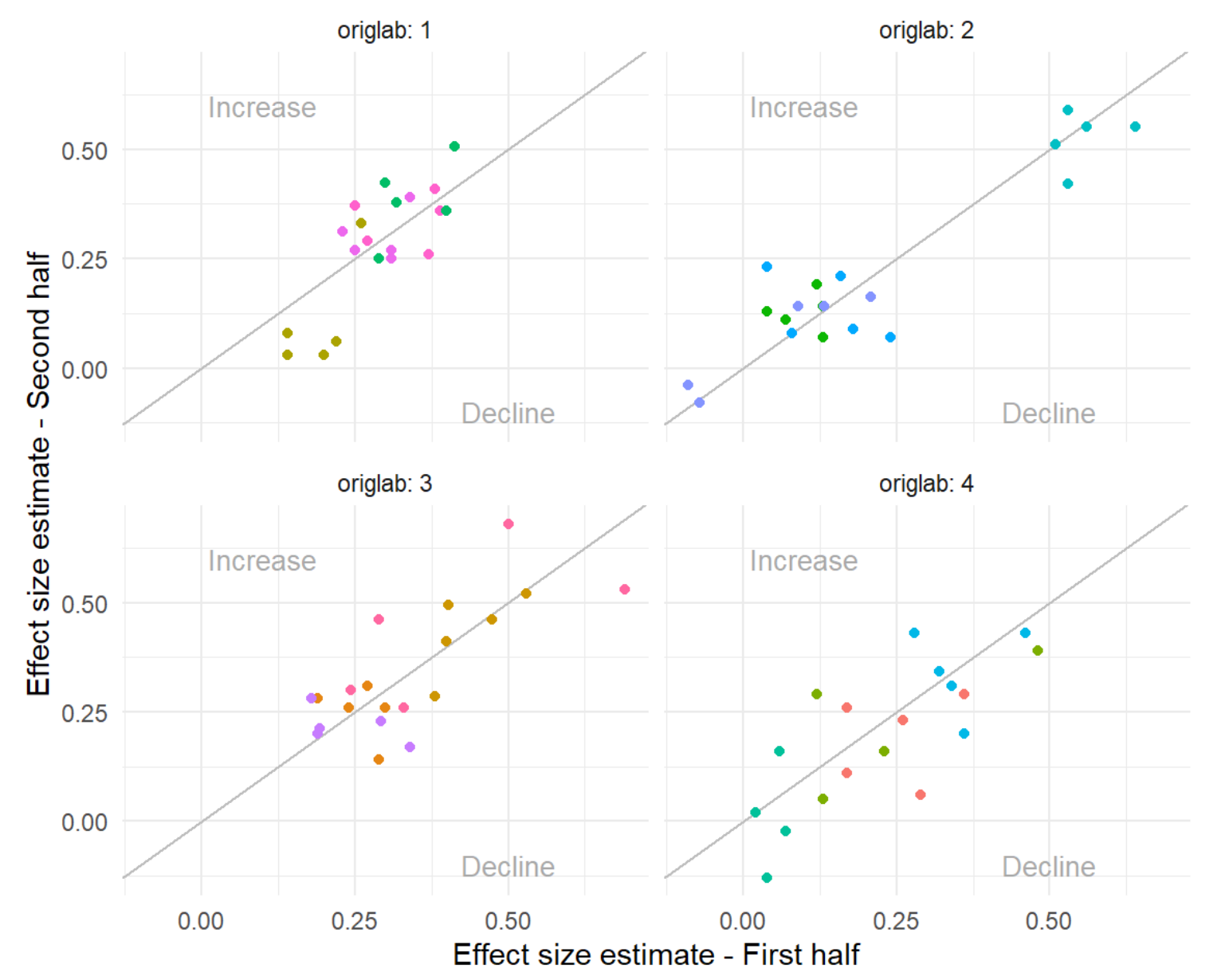

Fig S6: Effect size of the $1^{\text {st }} 750$ participants run vs. the effect size observed in the $2^{\text {nd }} 750$ participants within each lab. Participants were randomly assigned to take the study as part of the $1^{\text {st }}$ or $2^{\text {nd }} 750$, so this is exogenous temporal variation. Point estimates along the diagonal line correspond to observing the same effect size in both groups of participants.

To formally test these predictions, we estimated the parameters of a meta-regression equation that included terms for the sample half, the order of analysis, and their interaction. Let $H_{h i j k}=1 / 2$ when $h=2$ and $H_{h i j k}=-1 / 2$ when $h=1$. We estimated the parameters of the following meta-regression equation using the data from both halves of the confirmation and replication experiments from all 16 confirmatory tests:

$$
d_{h i j k}=\alpha_{k}+\beta_{1} H_{h i j k}+\beta_{2} A_{h i j k}+\beta_{3} H_{h i j k} A_{h i j k}+u_{j k}+v_{i j k}+e_{h i j k}
$$

where $\alpha_{k}$ is a fixed effect for each lab, representing the average effect size in studies originating from that lab, $\beta_{1}$ is the average change in effect size from first half to second half of the sample 
across experiments (the order of data collection effect), $\beta_{2}$ is the average difference in effect sizes between samples observed first and samples observed second (the order of observation effect), and $\beta_{3}$ represents the difference between the change in effect sizes between experiments where the first half was analyzed first and experiments where the first half was analyzed second (i.e., the interaction between the time effect and the observer effect). The equation also includes random effects for each study $\left(\mathrm{u}_{\mathrm{jk}}\right.$, for $\left.\mathrm{j}=1, \ldots, 4 ; \mathrm{k}=1, \ldots, 4\right)$ and experiment nested within study $\left(v_{\mathrm{ijk}}\right.$, for $\left.\mathrm{i}=0, \ldots, 4 ; \mathrm{j}=1, \ldots, 4 ; \mathrm{k}=1, \ldots, 4\right)$. The sampling error term $\mathrm{e}_{\mathrm{hijk}}$ is assumed to have known variance $\sigma_{\text {hijk }}^{2}$. We tested the hypothesis $\beta_{1}=0$ to examine time-based decline, $\beta_{2}=0$ to examine observer effects, and $\beta_{3}=0$ to examine the interaction. Hypothesis tests for $\beta_{1}$ and $\beta_{2}$ used testwise alpha levels of $\alpha=.025$ to control the family-wise error rate. The test of $\beta_{3}$ was treated as exploratory.

As a specification check for the tests of time-based decline and observer effects, we also estimated these effects using differences in effect sizes between sample halves. The main advantage of modeling the differences in effect sizes is that it requires weaker assumptions than fitting a model for the joint distribution of the effect size estimates.

For the test of time-based decline, let $\mathrm{d}_{-\mathrm{ijk}}=\mathrm{d}_{2 \mathrm{ijk}}-\mathrm{d}_{1 \mathrm{ijk}}$ denote the decline in effect sizes from the first half sample to the second half sample, with standard error calculated as $\sigma_{-i j k}=$ $\sqrt{\sigma_{1 i j k}^{2}+\sigma_{2 i j k}^{2}}$ Let $\mathrm{A}_{-\mathrm{ijk}}=\left(\mathrm{A}_{2 \mathrm{ijk}}-\mathrm{A}_{1 \mathrm{ijk}}\right)$, so that $\mathrm{A}_{-\mathrm{ijk}}=1$ if the first half sample was analyzed first and $\mathrm{A}_{-\mathrm{ijk}}=-1$ if the first half-sample was analyzed second. We estimated the following meta-analytic model:

$$
d_{-i j k}=\beta_{1}+\beta_{2} A_{-i j k}+u_{j k}+v_{i j k}+e_{i j k}
$$


where the sampling error term $\mathrm{e}_{\mathrm{ijk}}$ is assumed to have known variance $\sigma_{-i j k}^{2}$. The meta-regression coefficients have the same interpretation as in the previous model: $\beta_{1}$ is the average change in effect size from the first half to the second half of the sample and $\beta_{2}$ is the average difference in effect sizes between samples observed first and samples observed second. The random effects terms $\mathrm{u}_{\mathrm{jk}}$ and $\mathrm{v}_{\mathrm{ijk}}$ now capture study-level and experiment-level variation in the time-based decline, rather than variation in the original effect size estimates.

For the test of observer effects, we used the same approach as above, but based on the difference between effects observed first and those observed second. Let

$$
d_{A i j k}=2\left(A_{1 i j k} d_{1 i j k}+A_{2 i j k} d_{2 i j k}\right)
$$

with standard error given by $\sigma_{A i j k}=\sqrt{\sigma_{1 i j k}^{2}+\sigma_{2 i j k}^{2}}$. We estimated the following meta-analytic model:

$$
d_{A i j k}=\beta_{2}+\beta_{1} A_{-i j k}+u_{j k}+v_{i j k}+e_{i j k}
$$

where the sampling error term $\mathrm{e}_{\mathrm{ijk}}$ is now assumed to have known variance $\sigma_{A i j k}^{2}$. The meta-regression coefficients have the same interpretation as in the original model: $\beta_{2}$ is the average difference in effect size from the half-sample analyzed first to the half-sample analyzed second and $\beta_{1}$ is the average change in effect size from the first half to the second half of the sample. The random effects terms $u_{j k}$ and $v_{i j k}$ now capture study-level and experiment-level variation in the observer order effects, rather than variation in the original effect size estimates.

The effect size did not differ depending on whether participants were in the $1^{\text {st }} 750$ participants or the $2^{\text {nd }} 750$ participants $(b=-0.004, p=.658,95 \% \mathrm{CI}=-0.023$ to 0.015$)$, the order in which the data collected from those two groups were analyzed $(b=0.002, p=.789,95 \% \mathrm{CI}=-$ 0.016 to -0.021$)$, or the interaction between the two $(b=0.006, p=.867,95 \% \mathrm{CI}=-0.065$ to 
0.077). These results were the same when using the difference score between the two 750s of data collection and dropping the non-significant interaction term. Estimating the parameters of the equation with the difference between the $1^{\text {st }}$ and $2^{\text {nd }} 750$ as the DV showed the same null result $(b=0.002, p=.791,95 \% \mathrm{CI}=-0.016$ to 0.021$)$. The magnitude of effect was the same based on whether the $1^{\text {st }}$ or $2^{\text {nd }} 750$ was analyzed first $(b=-0.003, p=.663,95 \% \mathrm{CI}=-0.023$ to $0.015)$ 


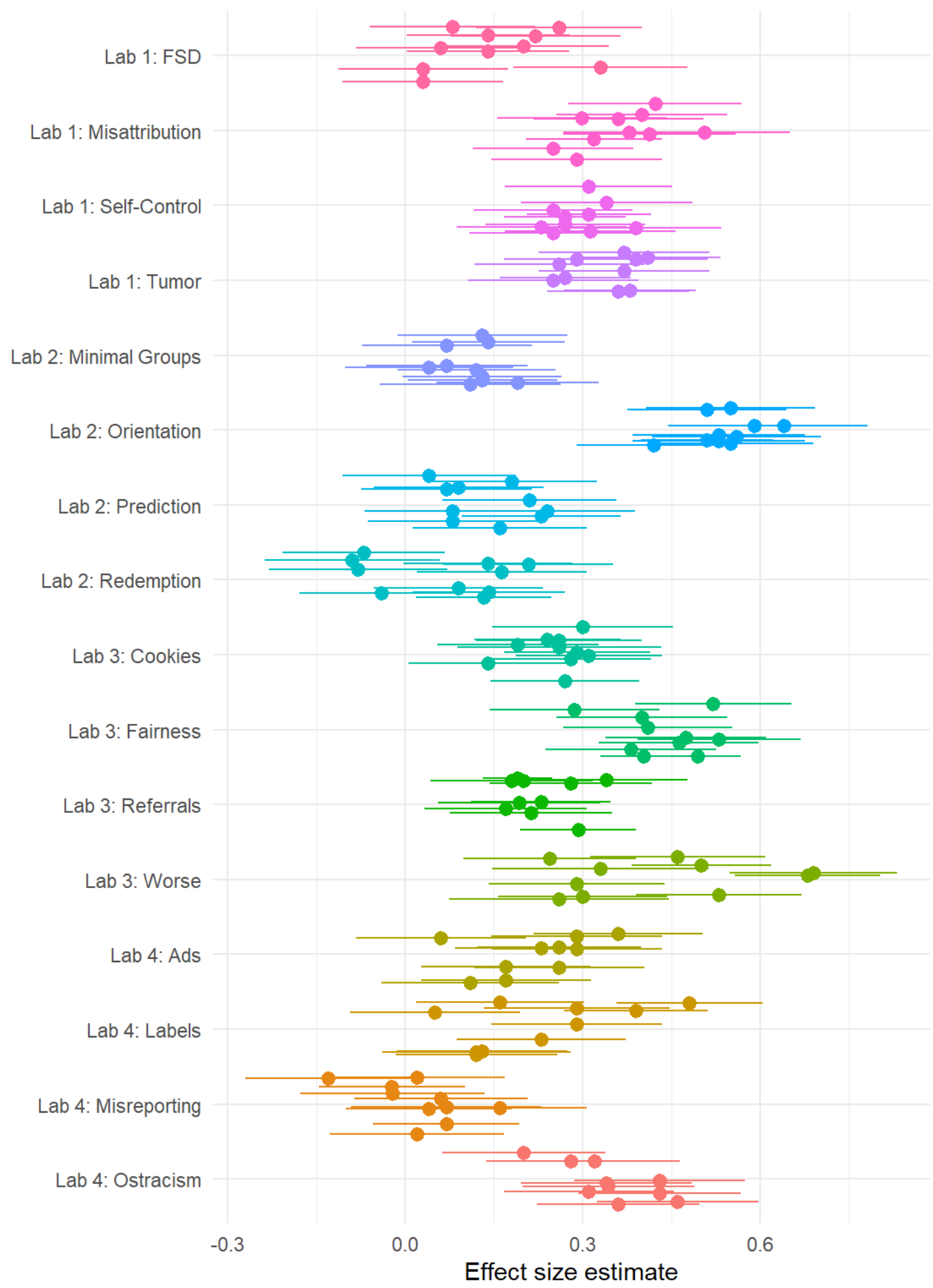

Fig S3: Effect size and 95\%CI from each 750/750 split replication of each effect. 


\section{Supplementary Information References}

S1. Open Science Collaboration. (2015). Estimating the reproducibility of psychological science. Science, 349(6251), aac4716. https://doi.org/10.1126/science.aac4716

S2. Klein, R., Ratliff, K., Vianello, M., Adams Jr, R., Bahník, S., Bernstein, M., ... \& Cemalcilar, Z. (2014). Data from investigating variation in replicability: A "many labs" replication project. Journal of Open Psychology Data, 2(1). https://doi.org/10.5334/jopd.ad

S3. Klein, R. A., Vianello, M., Hasselman, F., Adams, B. G., Adams Jr, R. B., Alper, S., ... \& Batra, R. (2018). Many Labs 2: Investigating variation in replicability across samples and settings. Advances in Methods and Practices in Psychological Science, 1(4), 443-490. https://doi.org/10.1177/2515245918810225

S4. Alogna, V. K., Attaya, M. K., Aucoin, P., Bahník, Š., Birch, S., Birt, A. R., ... \& Buswell, K. (2014). Registered replication report: Schooler and engstler-schooler (1990). Perspectives on Psychological Science, 9(5), 556-578.

S5. Ebersole, C. R., Mathur M., et al. (in principle acceptance). Many Labs 5: Testing pre-data collection peer review as an intervention to increase replicability. Advances in Methods and Practices in Psychological Science.

S6. Lipsey, M. W., \& Wilson, D. B. (2001). Practical meta-analysis. SAGE publications, Inc.

S7. Sánchez-Meca, J., Marín-Martínez, F., \& Chacón-Moscoso, S. (2003). Effect-size indices for dichotomized outcomes in meta-analysis. Psychological methods, 8(4), 448.

S8. Protzko, J., \& Schooler, J. W. (2017). Decline effects: Types, mechanisms, and personal reflections. Psychological science under scrutiny: Recent challenges and proposed solutions, 85107. https://doi.org/10.18637/jss.v036.i03

S9. R Core Team (2022). R: A language and environment for statistical computing. R Foundation for Statistical Computing, Vienna, Austria. https://www.R-project.org/.

S10. Viechtbauer, W. (2010). Conducting meta-analyses in R with the metafor package. Journal of Statistical Software, 36(3), 1-48. URL: http://www.jstatsoft.org/v36/i03/

S11. Pustejovsky, J. E. (2019). clubSandwich: Cluster-Robust (Sandwich) Variance Estimators with Small-Sample Corrections. R package version 0.3.5.

https://github.com/jepusto/clubSandwich

S12. Rouder, J. N., Speckman, P. L., Sun, D., Morey, R. D., \& Iverson, G. (2009). Bayesian t tests for accepting and rejecting the null hypothesis. Psychonomic bulletin \& review, 16(2), 225237. 